\title{
Experimental tests of QED in bound and isolated systems
}

\author{
Lucile JULIEN
}

QED 2012, Cargèse

3 lectures : 24, 25, 26 April 2012 


\section{The fine structure of atoms and the fine structure constant $\alpha$}

As the hyperfine structures, the fine structure frequencies vary as $\alpha^{2} R_{\infty}$

One can determine the $\mathrm{n}=2$ fine structure of hydrogen from the combination of optical measurements involving $2 \mathrm{P}$ states

The derived value of $1 / \alpha$ is : 137.036003(41) (accuracy $3 \times 10^{-7}$ )

It is not competitive since it is limited by the large natural width

of the $2 \mathrm{P}$ levels $(\sim 100 \mathrm{MHz})$

On another hand, in helium the $2^{3} \mathrm{P}$ states are narrower

(1.6 MHz)

since they cannot easily decay to the ground state

and the $2{ }^{1} \mathrm{~S}_{0}$ and $2{ }^{3} \mathrm{~S}_{1}$ states are metastable

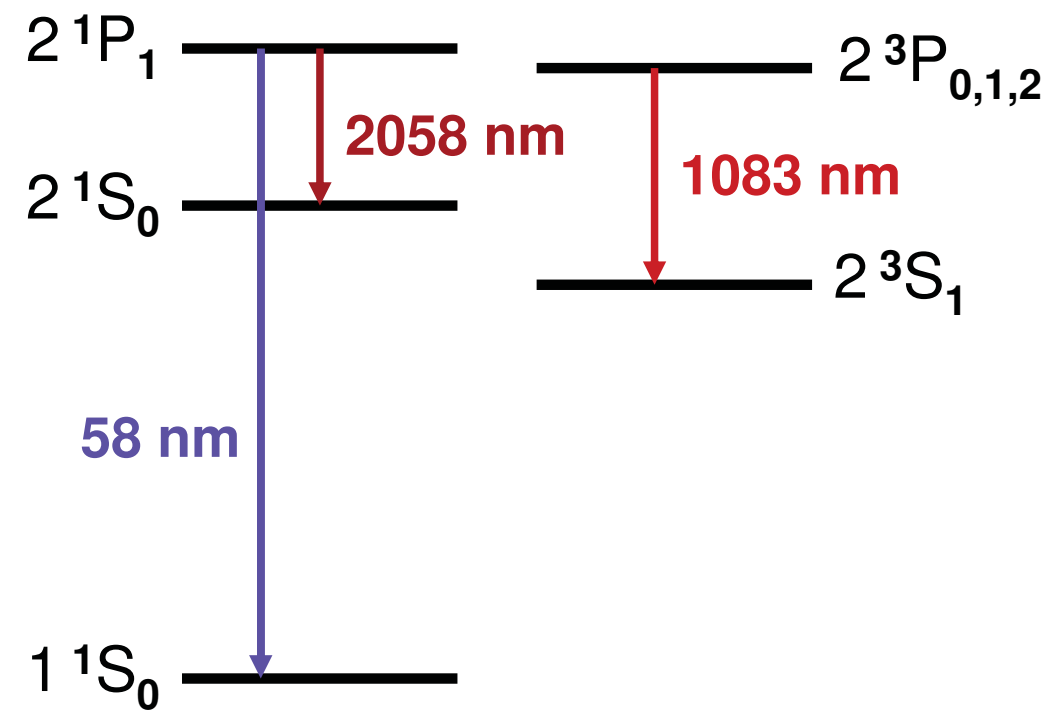




\section{The spectroscopy of helium atom : fine structure}

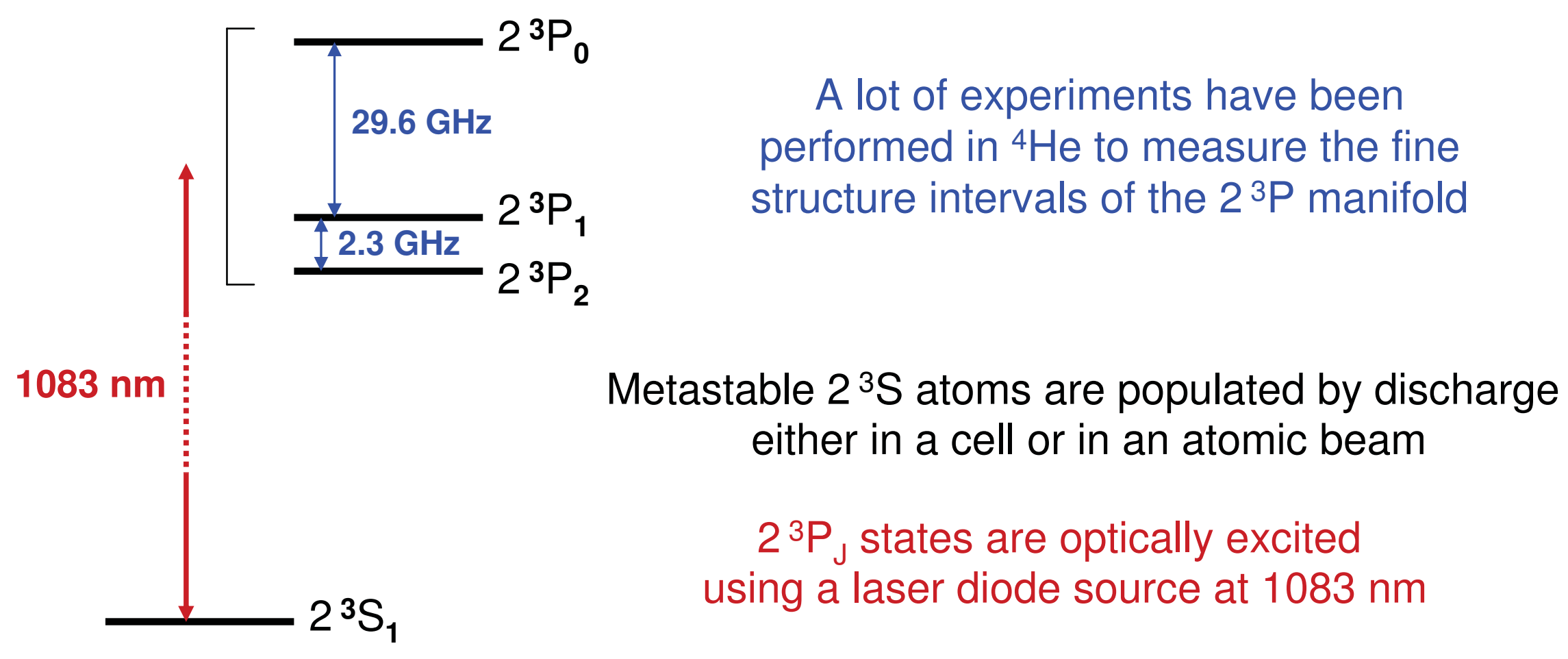

Frequency intervals have been determined either from optical line structures or by microwave techniques 


\section{The spectroscopy of helium atom : fine structure \\ The various experimental methods}

- Laser fluorescence technique with an atomic beam (LENS, Florence) combines sub-Doppler laser spectroscopy and direct microwave measurement

$$
\text { G. Giusfredi et al., Can. J. Phys. 83, } 301 \text { (2005) }
$$

- Separated oscillatory field microwave measurement of the $2{ }^{3} \mathrm{P}_{1}-2{ }^{3} \mathrm{P}_{2}$ interval uses an optical pulse in a thermal beam followed by two RF pulses (York U.)

$$
\text { J.S. Borbely et al., Phys. Rev. A 79, } 060503 \text { (2009) }
$$

- Doppler-free saturation spectroscopy in a cell (Harvard U.)

T. Zelevinsky, D. Farkas and G. Gabrielse, Phys. Rev. Lett. 95, 203001 (2005)

- Electro-optic laser technique in an atomic beam (North Texas U.)

uses modulated sidebands of a laser diode

to measure fine structure and various Zeeman intervals

M. Smiciklas and D. Shyner, Phys. Rev. Lett. 105, 123001 (2010)

All these measurements need very careful study of all systematic effects

(Zeeman, pressure, $2^{\text {nd }}$ order Doppler ...) 


\section{Helium fine structure : discussion}

All these accurate measurements of the fine structure of the $2^{3} \mathrm{P}$ level of helium are in good agreement each with others

They give a test of QED theory of the electron - electron interaction in bound systems

G.W.F. Drake and Z.-C. Yan, Can. J. Phys. 86, 45 (2008)

Until recently there were two inconsistent calculations

which disagreed with experimental results by $\sim 15 \mathrm{kHz}$ and more

Recent calculations up to $\alpha^{5} R_{\infty}$ terms

finally resolved this discrepancy

K. Pachucki and V.A. Yerokhin, Phys. Rev. Lett. 104, 070403 (2010)

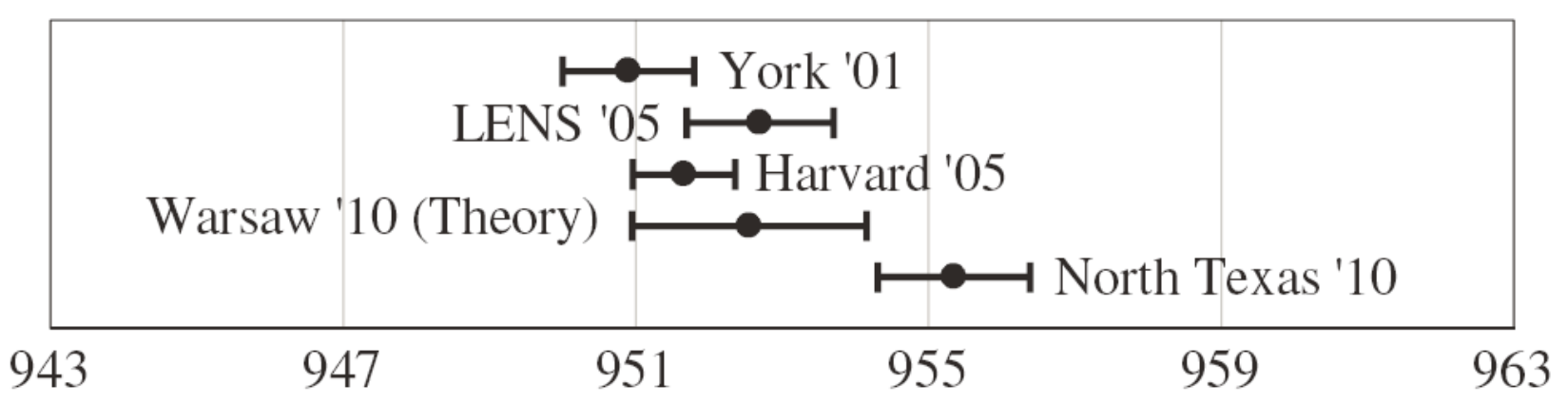

$2{ }^{3} P_{0}-2{ }^{3} P_{1}$ interval

$-29646000(\mathrm{kHz})$

from M. Smiciklas

and D. Shyner (2010)

If the validity of the theory is assumed, experimental fine structure measurements in helium give an independent determination of $\alpha$

with an uncertainty of $2 \times 10^{-8}$ mainly due to uncalculated high-order QED terms 


\section{Other spectroscopic measurements in helium}

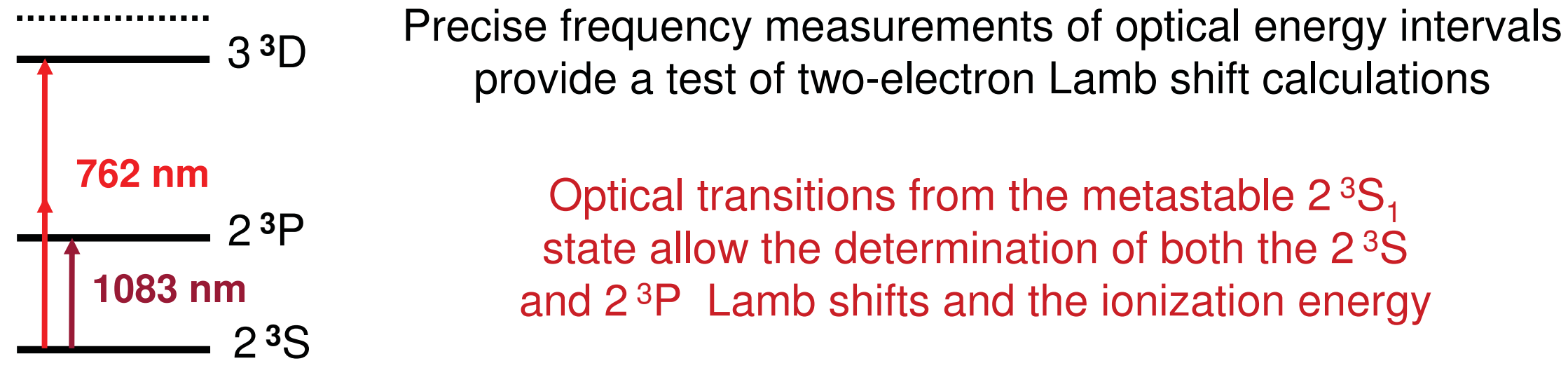

- Absolute frequency measurements of the $2{ }^{3} \mathrm{~S}_{1}-2^{3} \mathrm{P}_{0,1,2}$ transitions at $1083 \mathrm{~nm}$, with a frequency comb, give access to the $2^{3} \mathrm{~S}-2^{3} \mathrm{P}$ and $2^{3} \mathrm{P}$ Lamb shifts (Florence)

$$
\begin{aligned}
& f_{0}=276764094746.9(1.3) \mathrm{kHz} \\
& f_{1}=276734477805.0(0.9) \mathrm{kHz} \\
& f_{2}=276732186818.4(1.5) \mathrm{kHz}
\end{aligned}
$$

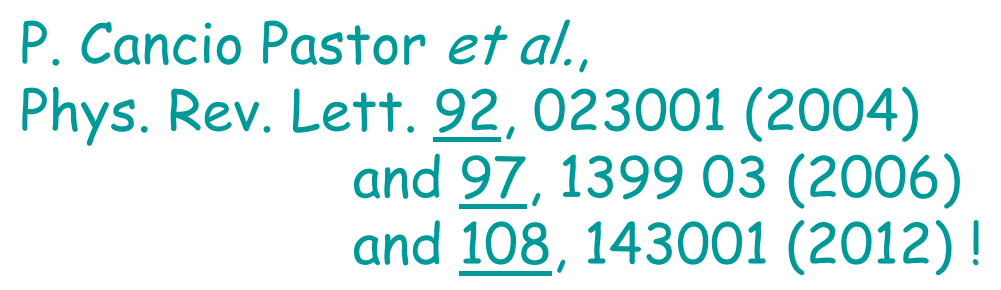

- Measurement of the two-photon $2{ }^{3} \mathrm{~S}_{1}-3^{3} \mathrm{D}_{1}$ transition at $762 \mathrm{~nm}$ (Paris) provides the ionization energy of the $2^{3} \mathrm{~S}$ state and a precise determination of the $2^{3} \mathrm{~S}$ Lamb shift through theoretical calculations on the $3{ }^{3} \mathrm{D}_{1}$ level

$$
f=786823850002(56) \mathrm{kHz} \quad \text { C. Dorrer et al., Phys. Rev. Lett. 78, } 3658 \text { (1997) }
$$




\section{Other spectroscopic measurements in helium}

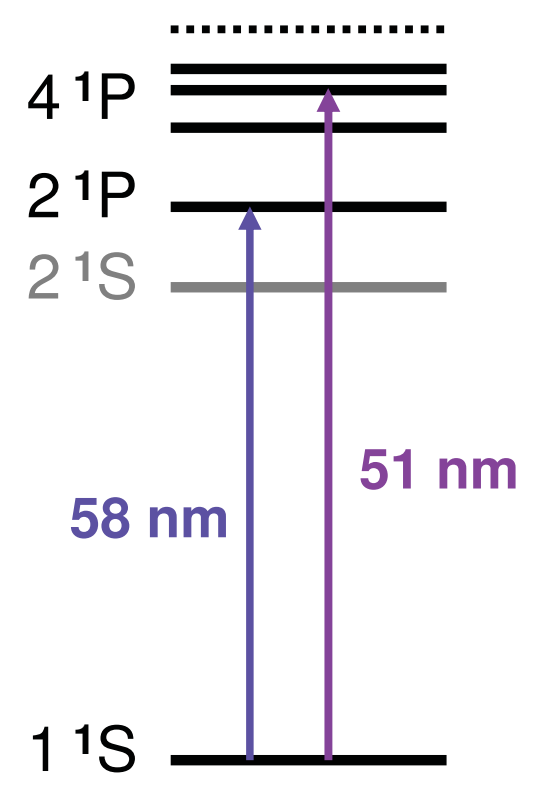

The recent development of UV laser sources and the extension of frequency combs in the UV range has open the way to the study of the resonance line of helium

- Measurement of the $1{ }^{1} \mathrm{~S}-2{ }^{1} \mathrm{P}$ resonance line at $58 \mathrm{~nm}$ (Amsterdam)

K.S.E. Eikema, W. Ubachs, W. Vassen and W. Hogervorst, Phys. Rev. A 드, 1866 (1997)

This experiment yields the $1^{1} \mathrm{~S}$ ground state Lamb shift

$$
\mathrm{L}_{1}=41224(45) \mathrm{MHz}
$$

to be compared to the calculated value 44233 (35) MHz

- Frequency comb metrology of the $1^{1} \mathrm{~S}-4^{1} \mathrm{P}$ and $1^{1} \mathrm{~S}-5^{1} \mathrm{P}$ lines (Amsterdam)

D.Z. Kandula et al., Phys. Rev. Lett. 105, 063001 (2010)

This experiment yields the ionization energy of ${ }^{4} \mathrm{He}$

- Metrology of the forbidden $2{ }^{3} S_{1}-2{ }^{1} S_{0}$ transition in cold atoms ... 


\section{Atomic spectroscopy with cold atoms}

We have already seen that the atomic frequency standard has been substantially improved by cooling and trapping the Cs atoms

More generally, the development of such techniques is of great interest for high resolution spectroscopy because of :

- the reduced Doppler effect

- the longer interaction time with light

- the advent of new derived physics

The basic idea is to use the recoil effect to reduce the atomic velocity
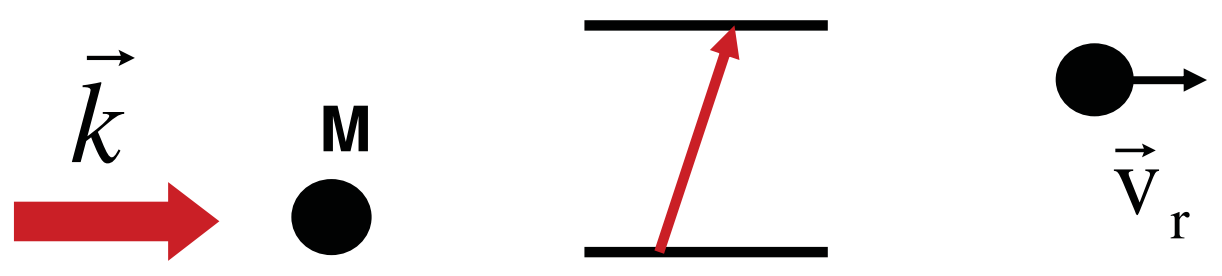

$$
\overrightarrow{\mathrm{v}}_{\mathrm{r}}=\frac{\hbar \vec{k}}{\mathrm{M}}
$$

Due to the absorption of a photon the atomic velocity is changed The recoil velocity is $3 \mathrm{~cm} / \mathrm{s}$ for a Na atom, since its thermal velocity is $400 \mathrm{~m} / \mathrm{s}$ First spectroscopic observation of recoil effect on a $\mathrm{CH}_{4}$ line :

J.L. Hall, Ch.J. Bordé, K. Uehara, Phys. Rev. Lett. 37, 1339 (1976) 


\section{Changing the atomic velocity}

The recoil effect is responsible for the radiation pressure

Since spontaneous emission is isotropic, it does not contribute to recoil

Recoil can be used :

- To deviate an atomic beam
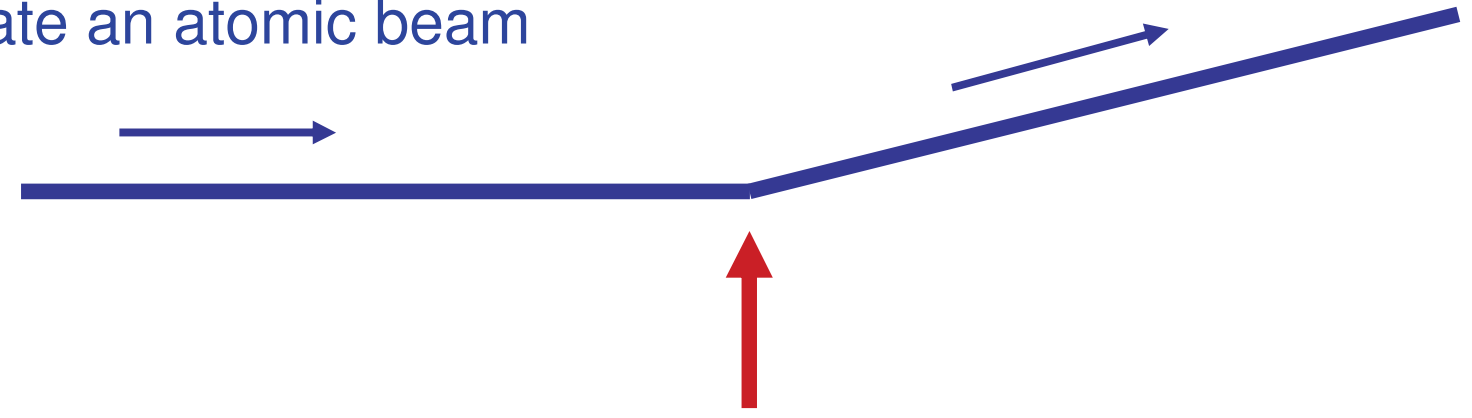

- To slow and stop an atomic beam
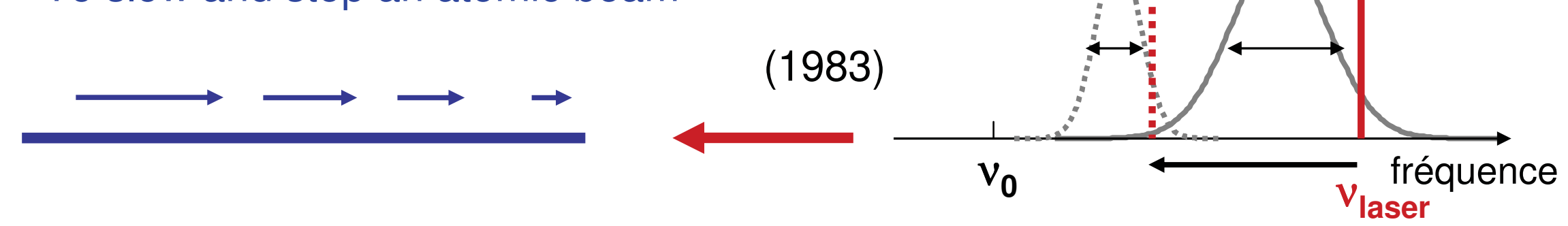

To keep atoms in resonance with the laser light during the slowing process, one must sweep the laser frequency or the atomic frequency (Zeeman effect)

The beam can be stopped on a $1 \mathrm{~m}$ distance 


\section{Laser cooling and trapping of atoms}

- Cooling : reduce the velocity in all directions

Atoms in a gas are enlighted by three pairs of laser beams (one in each direction of space)

which frequency is slightly lower than $v_{0}$

Due to Doppler effect, each atom absorbs

more the beam which is counterpropagating

with its velocity and then is slowed down

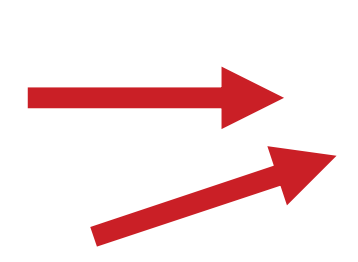

Optical molasses

$2 \mathrm{~cm} / \mathrm{s} \sim 4 \mu \mathrm{K}$

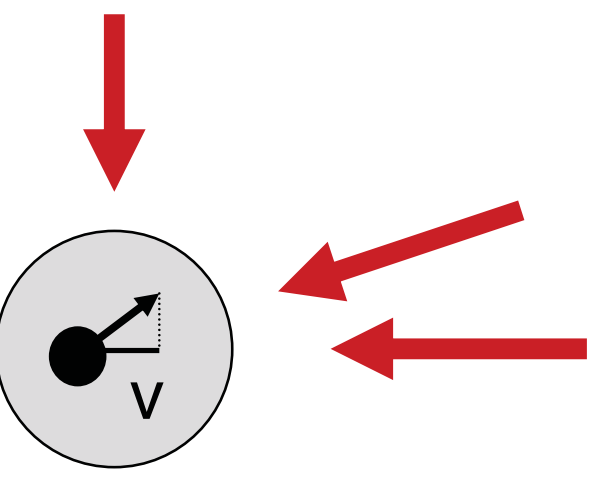

- Trapping : confine the atoms in a given region of space

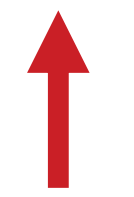

Magneto-optical trap (MOT)

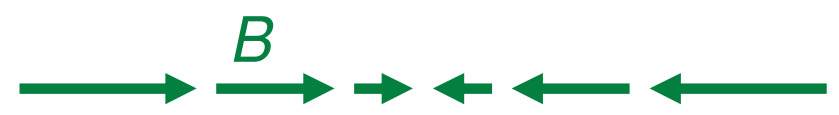

A spatially varying magnetic field is added to cooling polarized laser beams

to induce a minimum Zeeman shift in the center of the trap
Dipole trap

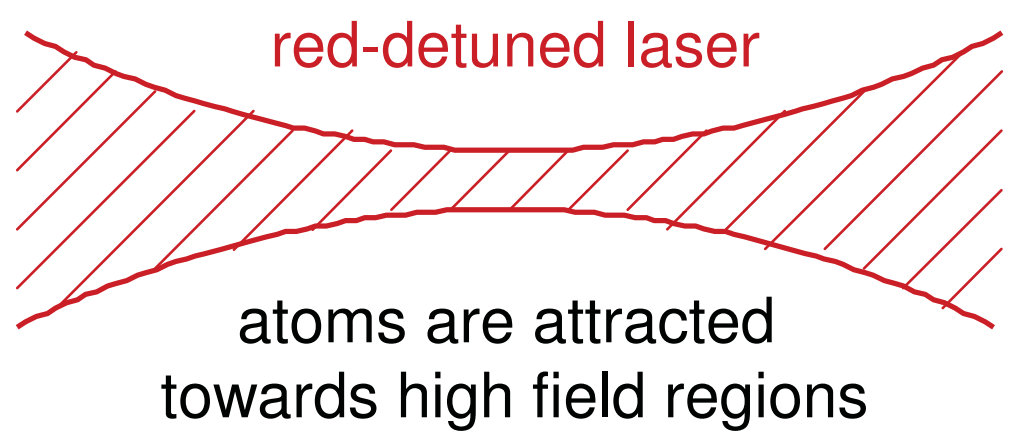


Lower and lower temperatures (300 nK) by evaporating cooling ....

... and high cold atom density by efficient trapping

Quantum degeneracy is reached when the De Broglie wavelength is of the same order of magnitude as the distance between atoms

$$
n \lambda_{D B}^{3} \approx 1
$$

For bosonic atoms, one can then observe Bose-Einstein condensation

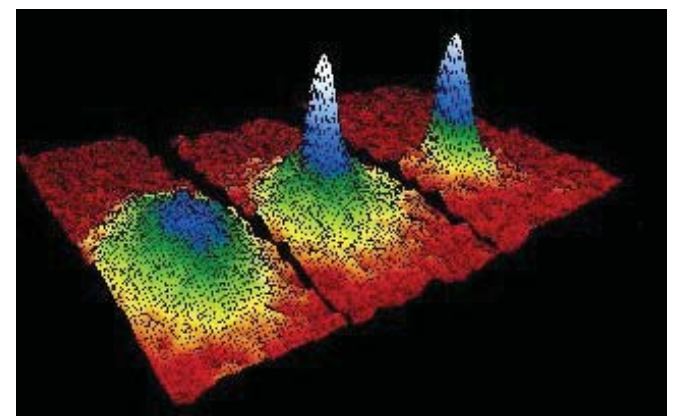

velocity distribution

(Rb 1995)

BEC has been presently obtained in various atoms :

$\mathrm{Na}, \mathrm{K}, \mathrm{Rb}, \mathrm{Cs}, \mathrm{H}, \mathrm{He}^{\star}, \mathrm{Sr}, \mathrm{Yb}, \mathrm{Cr}$... 


\section{Frequency metrology in quantum degenerate helium}

Helium in the $2^{3} S_{1}$ state can be laser-cooled and trapped allowing much longer interaction time with a laser beam and then excitation of a weak transition
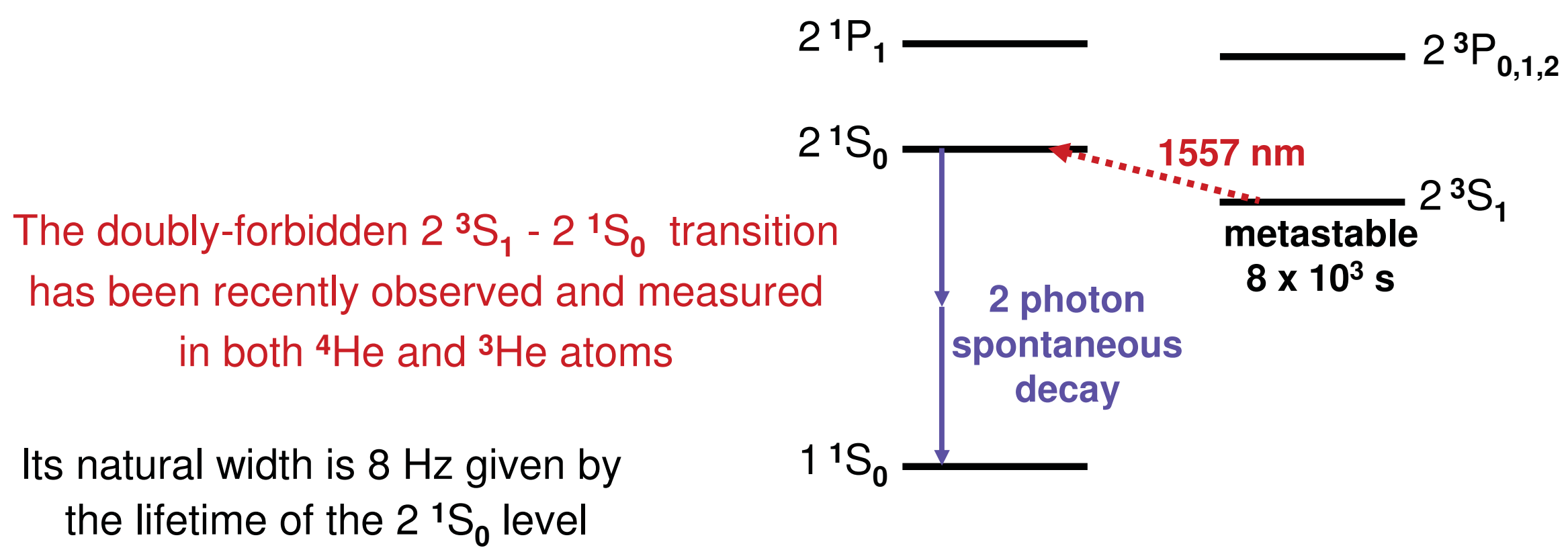

This gives a sensitive test of QED calculations and nuclear structure effects

$$
\text { R. van Rooij et al., Science 333, } 196 \text { (2011) }
$$




\section{Frequency metrology in quantum degenerate helium}

\section{Experimental set-up}

$2{ }^{3} \mathrm{~S}_{1}$ atoms are produced by electron impact in a discharge

They are slowed and trapped by laser techniques at $1083 \mathrm{~nm}$

- ${ }^{4} \mathrm{He}^{\star}$ are evaporatively cooled toward Bose Einstein condensation

- ${ }^{3} \mathrm{He}^{*}$ reach quantum degeneracy by sympathetic cooling with ${ }^{4} \mathrm{He}^{*}$

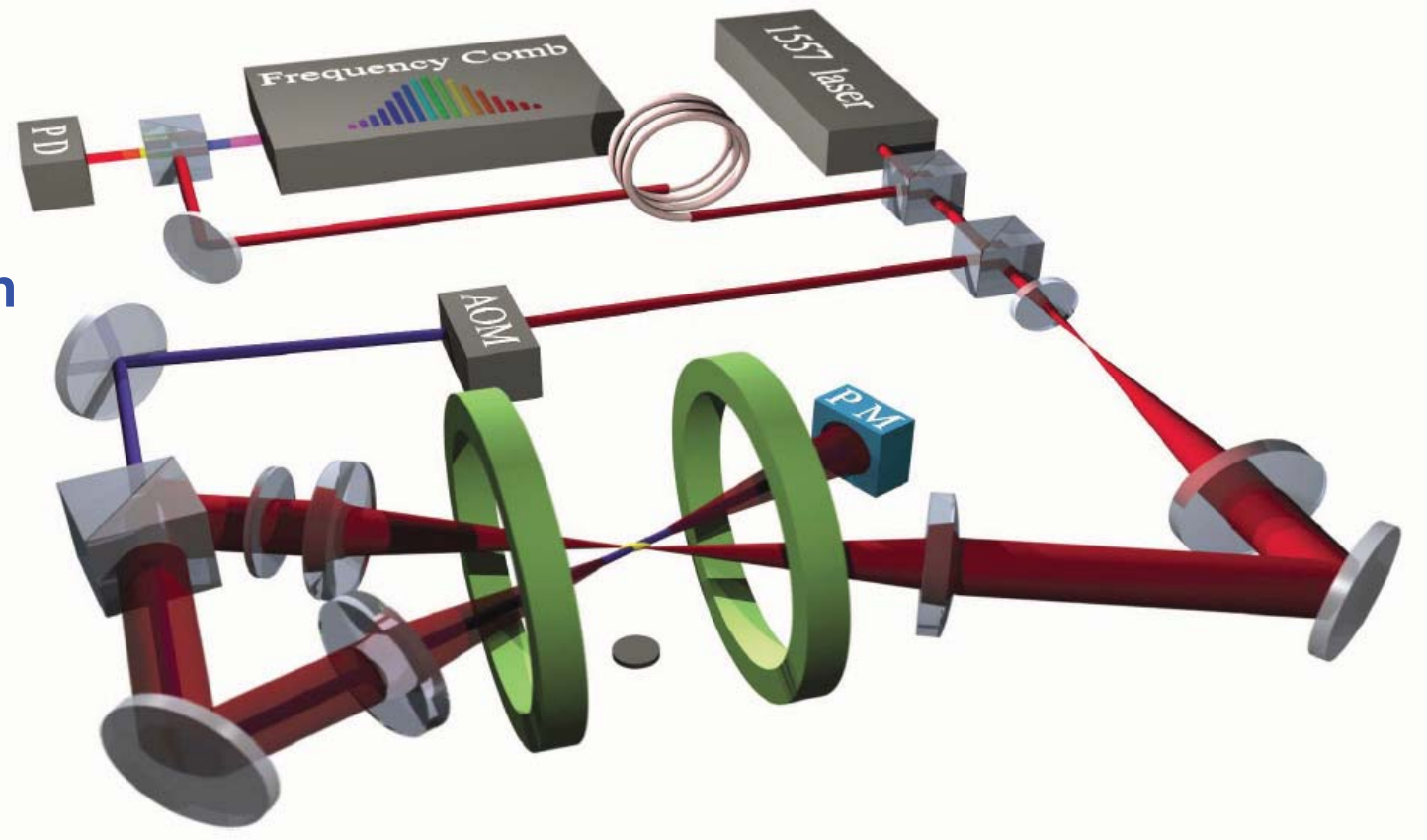

$\sim 10^{6}$ ultracold atoms are transferred into a crossed beam optical dipole trap

where they are illuminated by a resonant light at $1557 \mathrm{~nm}$

After free falling, remaining atoms in the $2{ }^{3} S_{1}$ state are detected by a microchannel plate detector 


\section{Frequency metrology in quantum degenerate helium}

\section{Signals}

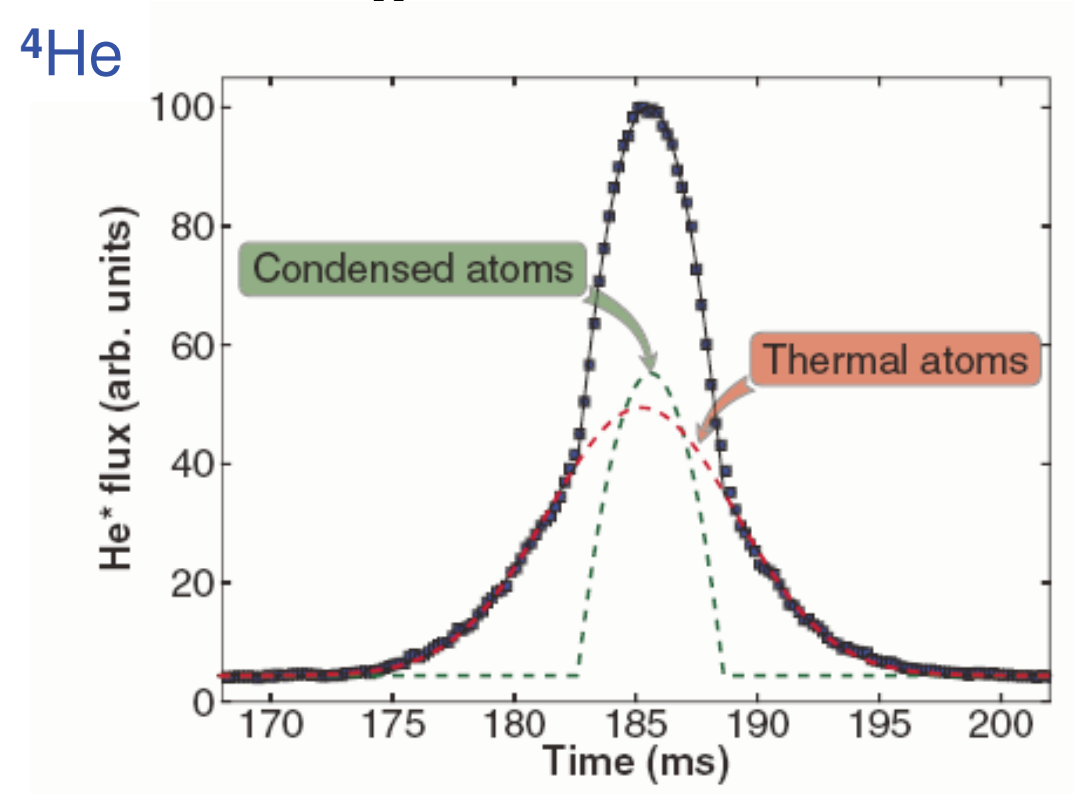

bimodal time-of-flight distribution

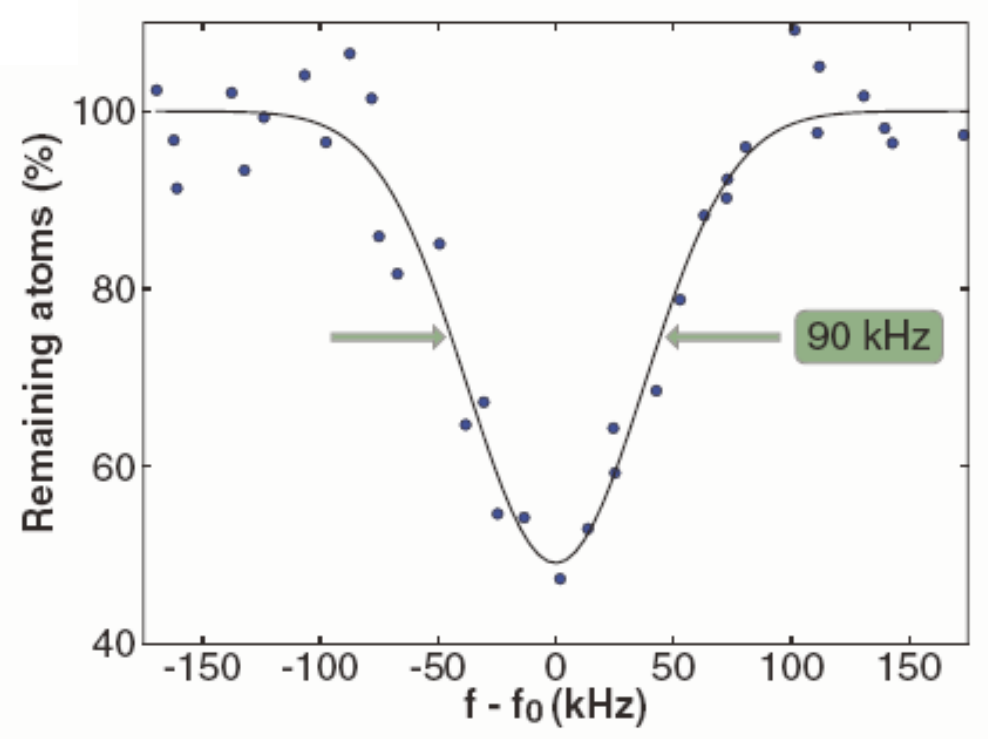

Gaussian fit of the line profile

\section{Results}

Frequencies measured with a frequency comb

- in ${ }^{4} \mathrm{He}: \mathrm{f}_{4}=192510702145.6(1.8) \mathrm{kHz}$

- in ${ }^{3} \mathrm{He}: \mathrm{f}_{3}=192504914426.4(1.5) \mathrm{kHz}$

This experiment also allows to deduce the isotope shift 


\section{The spectroscopy of helium atom : conclusion}

During the last years, various accurate frequency measurements have been performed in helium :

- Fine structure intervals in the $2^{3} \mathrm{P}$ state

- Optical transitions from the $2^{3} S$ metastable state

- UV transitions from the $1{ }^{1} \mathrm{~S}$ ground state

- Inter-combination $2{ }^{3} S-2{ }^{1} S$ line

- Hyperfine structure of ${ }^{3} \mathrm{He}$ and isotope shift (non discussed here)

and significant advances have been accomplished in theoretical calculations so that the level of precision is approaching the experimental one

(except for the 2S Lamb shift)

The recent experiments provide :

- the fine and hyperfine structures

- the binding energies

- the Lamb shifts

and then allow to test electron - electron interaction and QED effects

in this 3-body atomic system 


\section{Experimental tests of QED in bound systems}

Already discussed:

Spectroscopy of hydrogen and helium atoms $(Z=1$ and 2$)$

including experimental methods :

- microwave measurements

- high resolution laser spectroscopy

- metrology of optical frequencies

- cold atoms techniques

Lamb shift measurements give stringent test of QED calculations but are limited by the knowledge of nuclear charge radius

In the following of this lecture :

- We will see if it is possible to overcome this limitation

- by the study of pure leptonic atomic systems

- or by the determination of the proton radius

- And we will consider highly charged ions to test QED corrections in high Z systems 


\section{The positronium $\mathrm{e}^{+}-\mathrm{e}^{-}$}

It is the lightest exotic hydrogen-like atom and a purely leptonic sytem and allows to test relativistic two-body and QED corrections

Electron and positron have same mass, same spin, but opposite charges

Energetic positrons are produced by radioactive sources

Positronium can be formed :

- by stopping energetic positrons in a gas or in a powder

- or by charge exchange of slow positrons in a thin foil or a gas target

- or by interaction of slow positrons with the surface of a solid in vacuum

The $\mathrm{e}^{+}-\mathrm{e}^{-}$annihilation is responsible for specific terms in energy levels and limits the lifetimes 


\section{Energy levels and lifetimes in positronium}

$2^{1} \mathrm{P}_{1}$
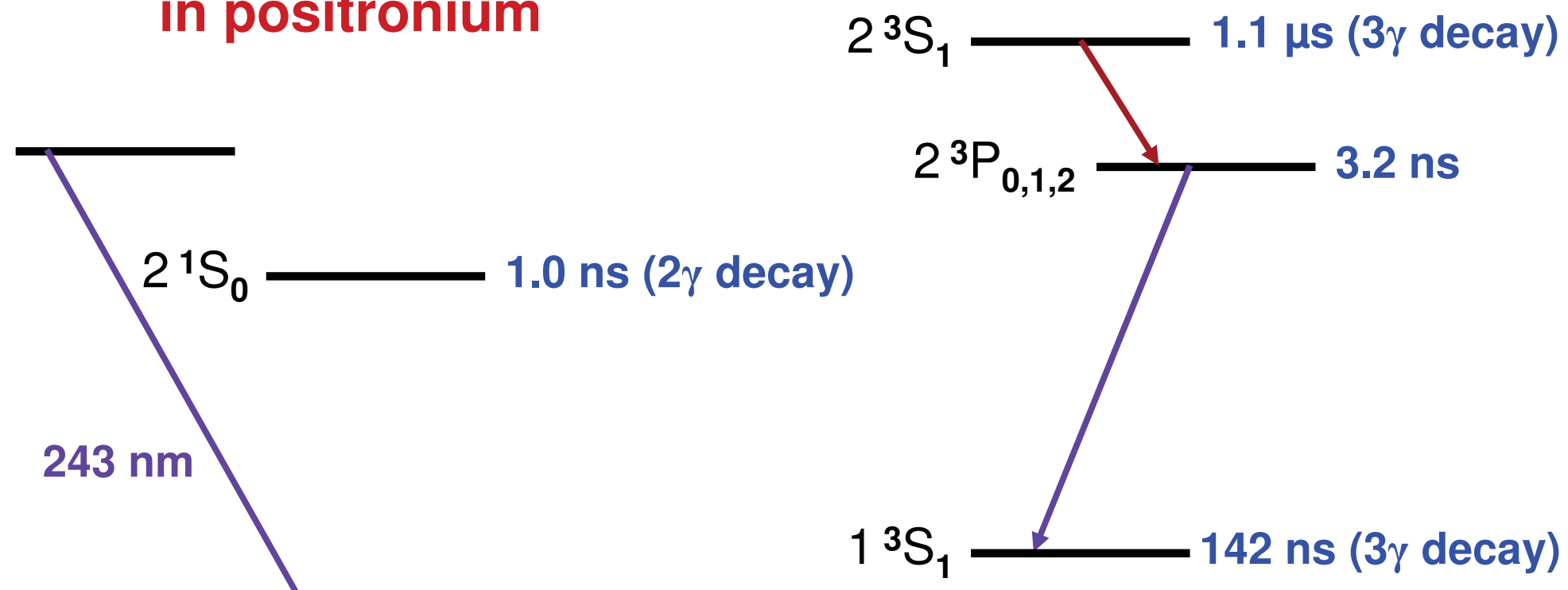

$1^{1} \mathrm{~S}_{0}$

$0.125 \mathrm{~ns}(2 \gamma$ decay)

Parapositronium (singlets)

Orthopositronium (triplets)

Lifetimes are much longer in orthopositronium

Because of present precision in theory and experiments, the experimental data which can be used to test QED predictions in positronium are :

- the annihilation rates in triplet and singlet ground states

- the $\mathrm{n}=1$ hyperfine interval

- the $\mathrm{n}=2$ fine structure interval

- the 1S-2S triplet interval 


\section{Lifetimes of ortho- and para- positronium in their ground states}

They result from $\mathrm{e}^{+}-\mathrm{e}^{-}$annihilation

Because of parity conservation, the decay process is different :

$2 \gamma$ decay for the singlet $(0.125 \mathrm{~ns})$

$3 \gamma$ decay for the triplet (142 ns)

A series of measurements have been performed ( $U$. of Michigan) determining

the decay rate of a positronium beam either in a gas or in vacuum

D.W. Gidley, A. Rich, E. Sweetman and D. West, Phys. Rev. Lett. 49, 525 (1982)

C.I. Westbrook, D.W. Giley, R.S. Conti and A. Rich, Phys. Rev. Lett. 58, 1328 (1987)

J.S. Nico, D.W. Gildey and A. Rich, Phys. Rev. Lett. $\underline{65}, 1344$ (1990)

Extensive efforts have been carried out to more and more improve the precision and check all the sytematic effects

In 1990, the results were :

$$
\begin{aligned}
& \lambda_{\mathrm{S}}=7.994(0.011) \mathrm{ns}^{-1} \text { in agreement with theoretical prediction } \\
& \lambda_{\mathrm{T}}=7.0516(0.0013) \mu \mathrm{s}^{-1} \text { (in gas) } \\
& \lambda_{\mathrm{T}}=7.0482(0.0016) \mu \mathrm{s}^{-1} \text { (in vacuum) }
\end{aligned}
$$

Long standing discrepancy by more than $6 \sigma$ with theoretical prediction on $\lambda_{T} \ldots$ 
... now resolved!

R.S. Vallery, P.W. Zitewitz and D.W. Gidley, Phys. Rev. Lett. 90, 203402 (2003)

(Michigan)

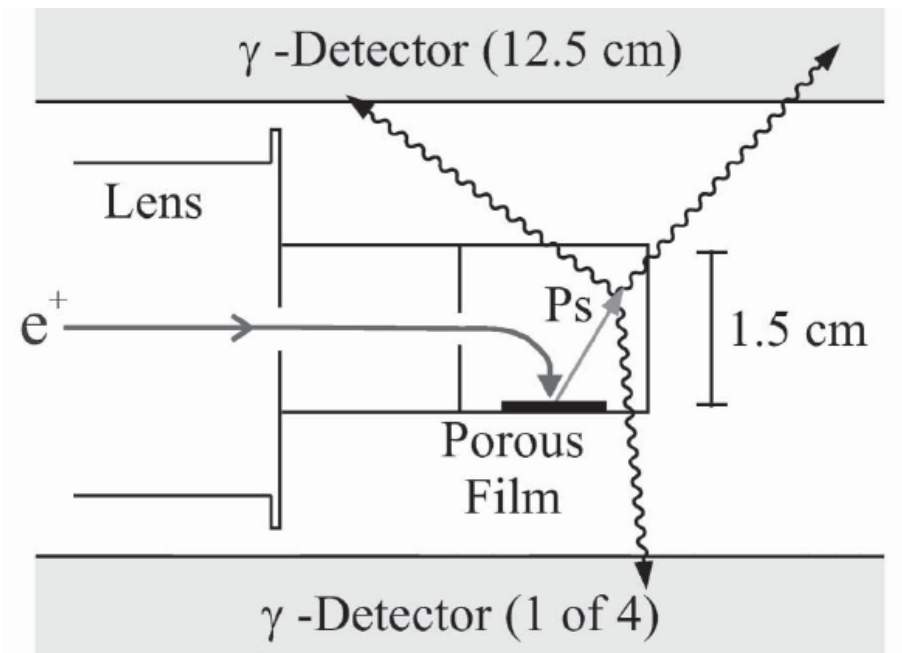

Ps is formed in a porous silica film in near thermal equilibrium and confined in a small cavity having negligible wall quenching

The spectrum is corrected from backscattering due to high energy Ps

The result is : $\lambda_{T}=7.0404(10)(8) \mu s^{-1}$

in agreement with theory : $\lambda_{T}=7.039979(11) \mu \mathrm{s}^{-1}$

G.S. Adkins, R.N. Fell and J. Sapirstein,

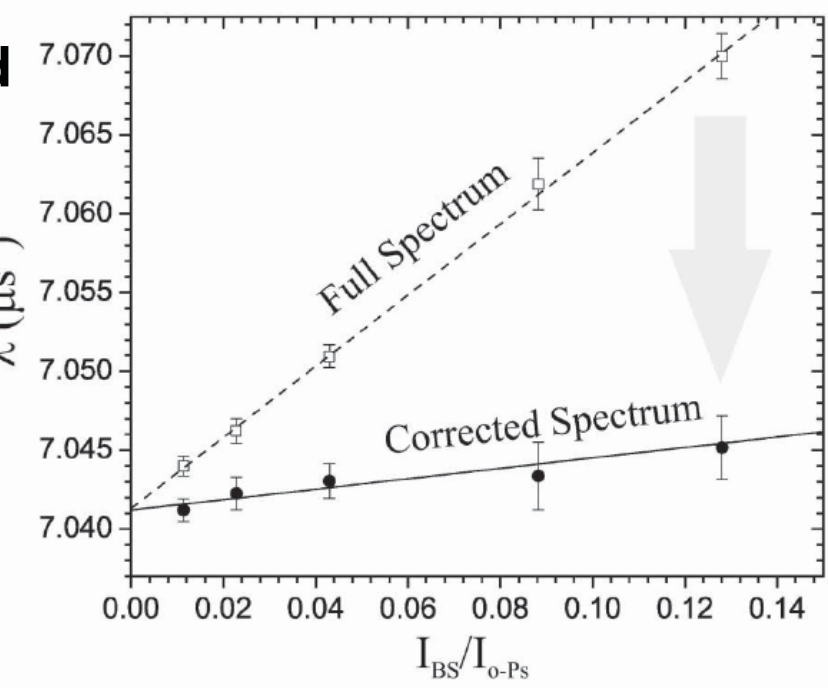

Ann. Phys. (N.Y.) 295, 136 (2002)

Another recent measurement performed in Tokyo confirms this excellent agreement

Y. Kataoka, S. Asai and T. Kobayashi, Physics Letters B 671, 219 (2009)

The combined experimental result is now : $\lambda_{\mathrm{T}}=7.0401(7) \mu \mathrm{s}^{-1}$

giving a good test of validity for the second order $O\left(\alpha^{2}\right)$ corrections 


\section{The hyperfine structure of the ground state of positronium}
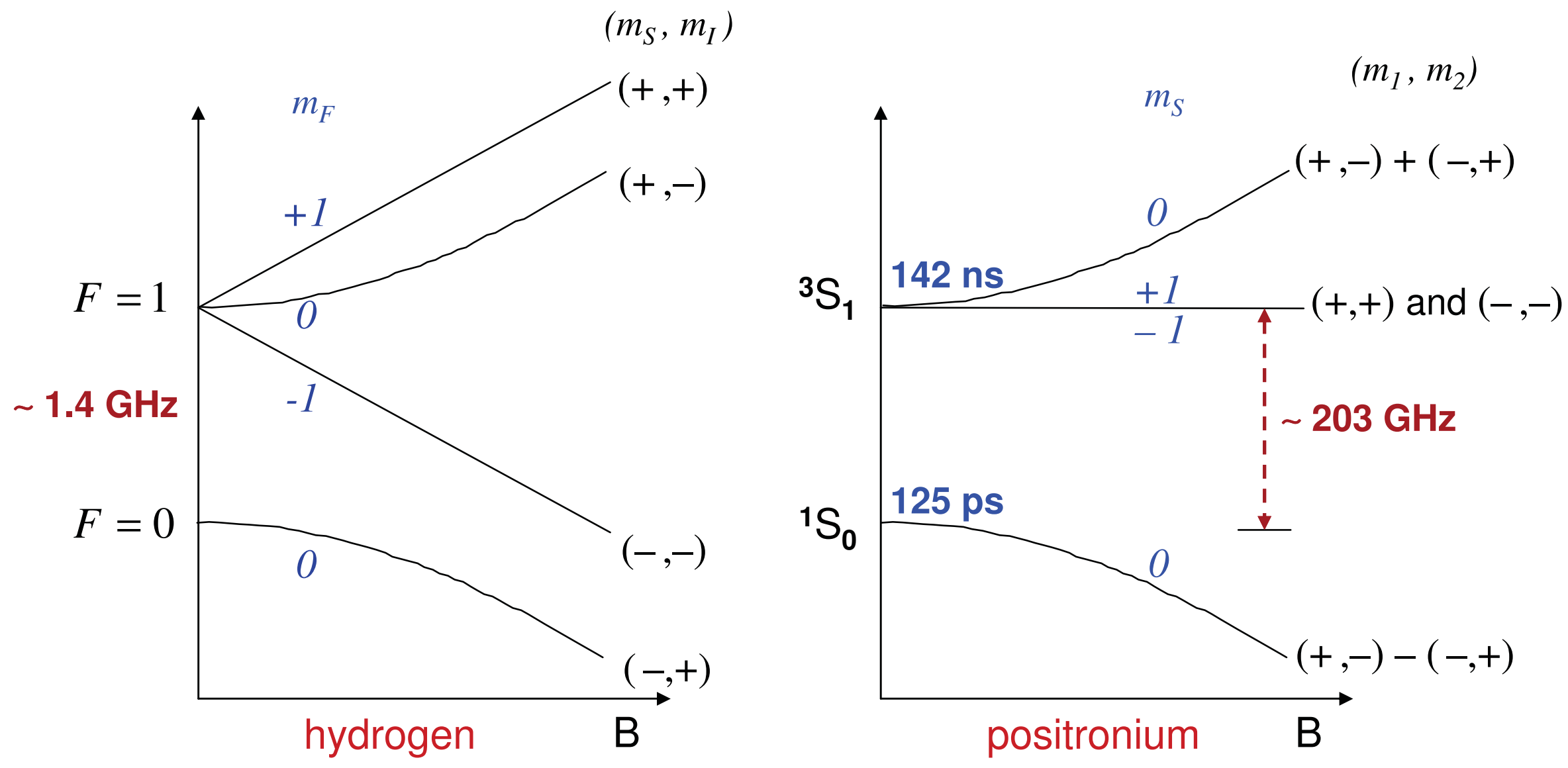

$\mathrm{e}^{+}$and $\mathrm{e}^{-}$have opposite gyromagnetic ratios so that $(+,+)$ and $(-,-)$ states have null magnetic momentum 
Measurement of the hyperfine structure of positronium through the Zeeman splitting

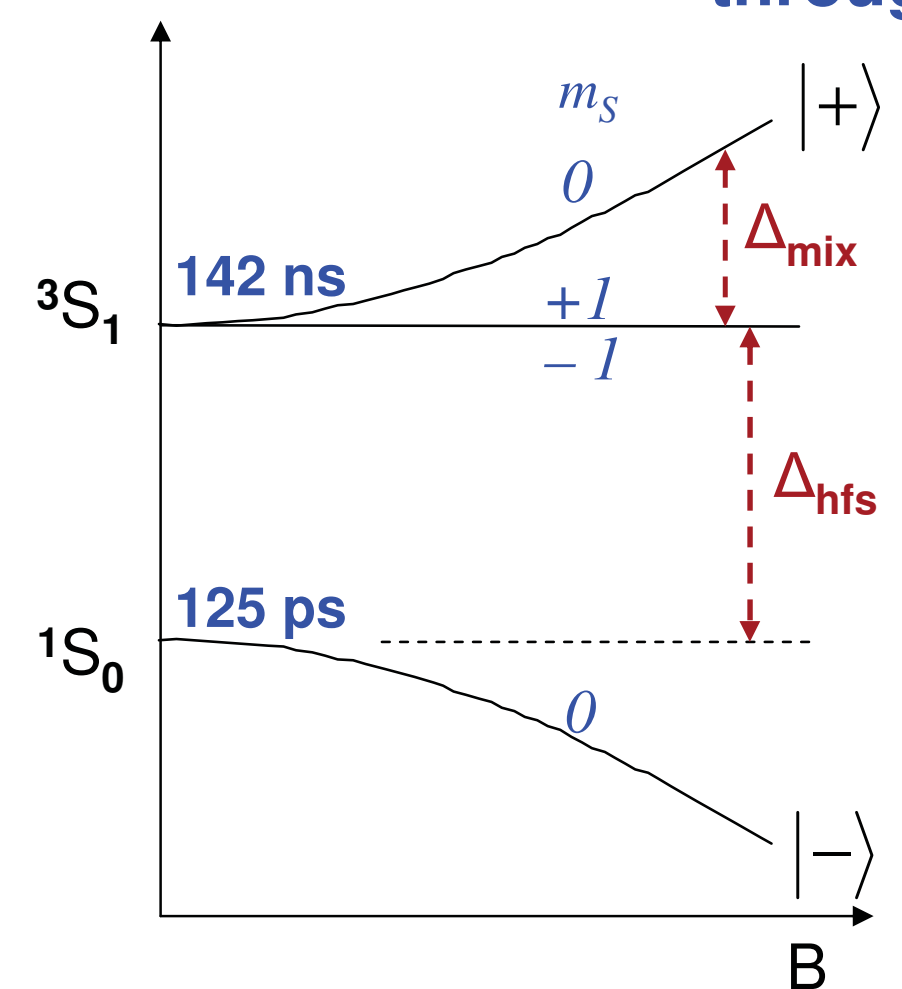

- Quantum oscillation Y. Sasaki and al. , (Tokyo) Physics Letters B 697, 121 (2011) Result : $\Delta_{\text {hfs }}=203.324$ (39) $\mathrm{GHz}$ less precise but could be improved

- and also direct $\mathrm{THz}$ excitation

T. Yamazaki et al., arXiv (5 Apr. 2012)
$\Delta_{\text {mix }}$ is related to $\Delta_{\text {hfs }}$ by a simple formula Two approaches :

- Microwave excitation at frequency $\Delta_{\text {mix }}(\sim 3 \mathrm{GHz}$ in $0.8 \mathrm{~T}$ ) followed by an increase of $2 \gamma$ decay M.R. Ritter et al., Phys. Rev. A 30, 1331 (1984) Result : $\Delta_{\text {hfs }}=203.38910$ (74) GHz (Yale) (3.9 $\sigma$ from theory)

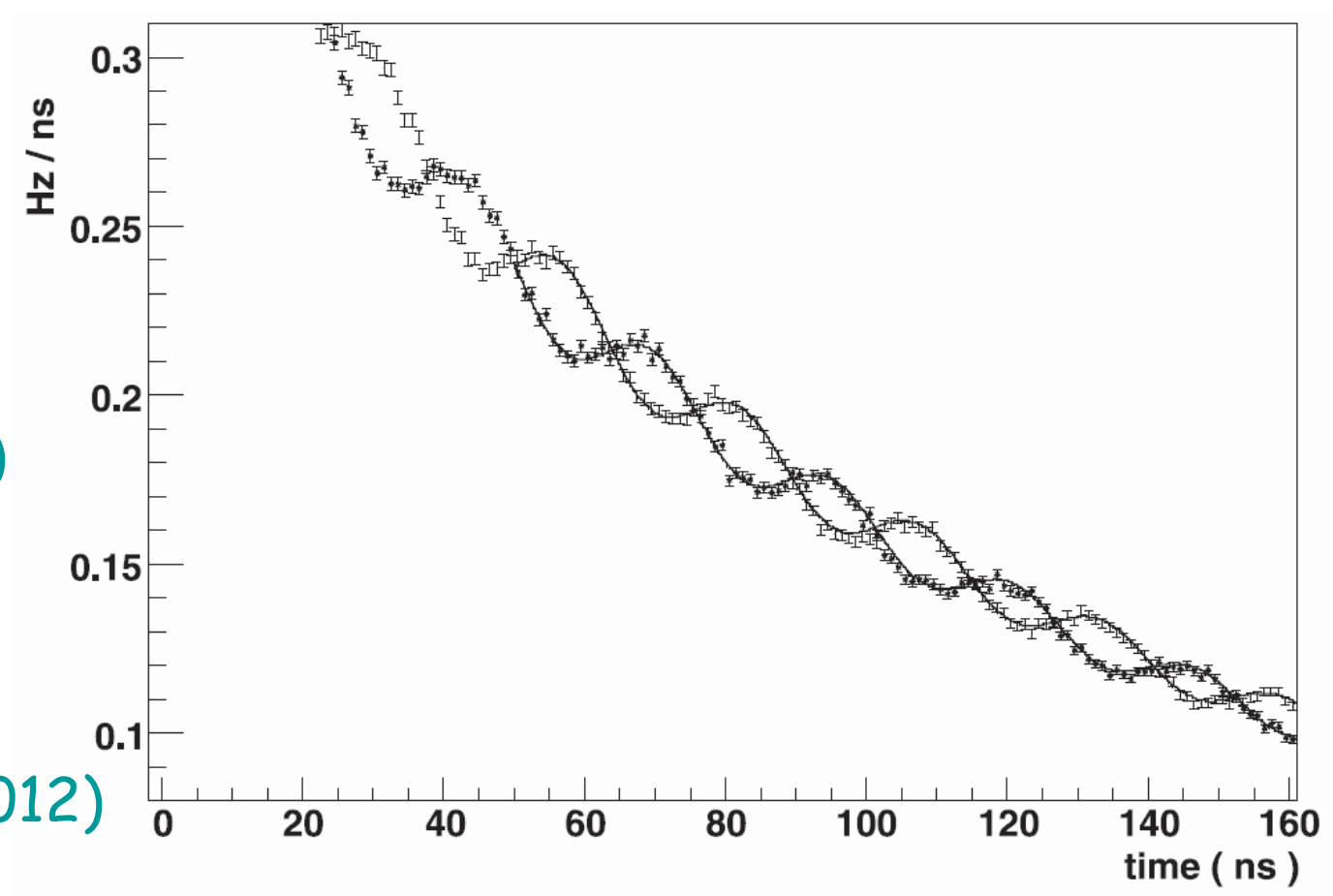


Measurements of the $\mathbf{n}=\mathbf{2}$ fine structure intervals in positronium

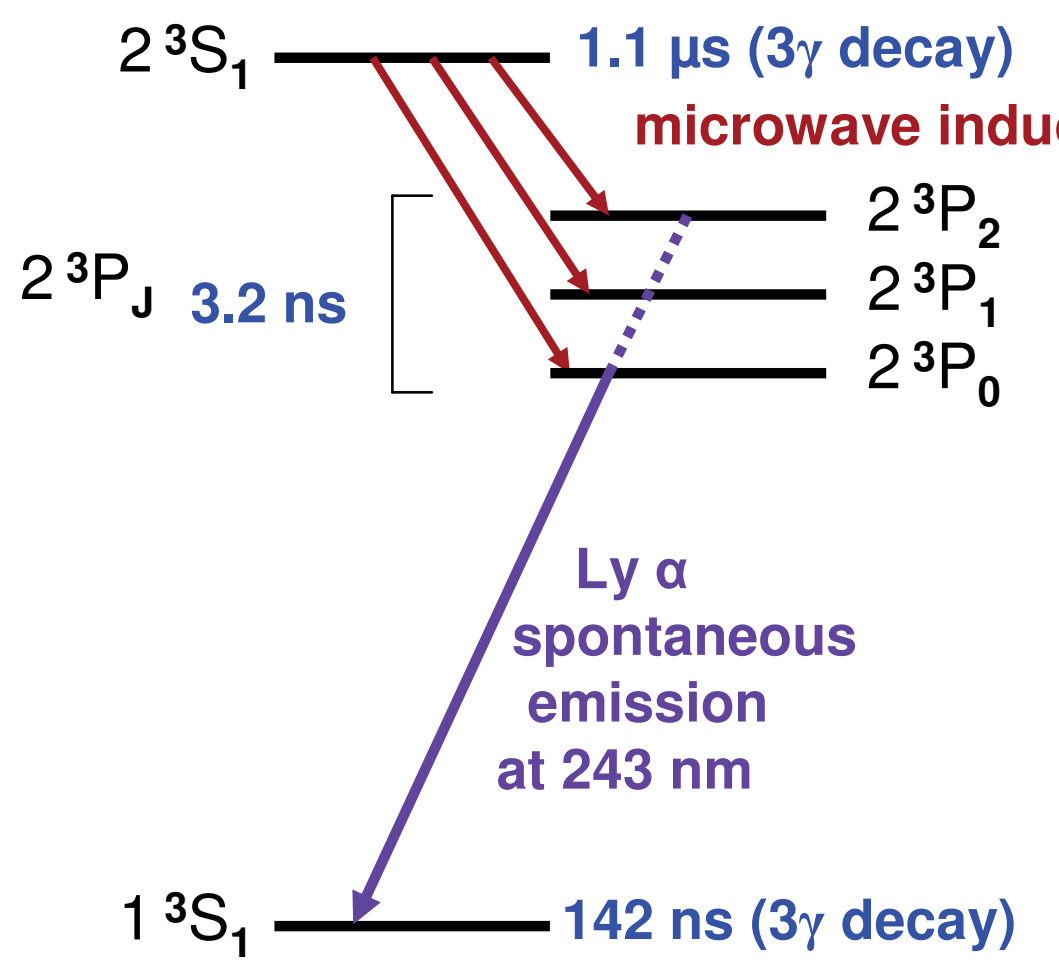

$2^{3} \mathrm{~S}_{1}$ and $2^{3} \mathrm{P}_{\mathrm{J}}$ states are all populated when positronium atoms are formed

The microwave transitions are detected through the increase of Ly a fluorescence

The measured frequencies are :

$$
\begin{aligned}
& v_{0}=18499.65(1.20)(4.00) \mathrm{MHz} \\
& v_{1}=13012.42(0.67)(1.54) \mathrm{MHz}
\end{aligned}
$$$$
\text { (Mainz) } \quad v_{2}=8624.38(0.54)(1.40) \mathrm{MHz}
$$

D. Hagena, R. Ley, D. Weil and G. Werth, Phys. Rev. Lett. 71, 2887 (1993)

Agreement with recent calculated QED corrections in $R_{\infty} \alpha^{4} \ln \alpha^{-1}$ 
Frequency measurement of the $1^{3} S_{1}-2{ }^{3} S_{1}$ transition of positronium

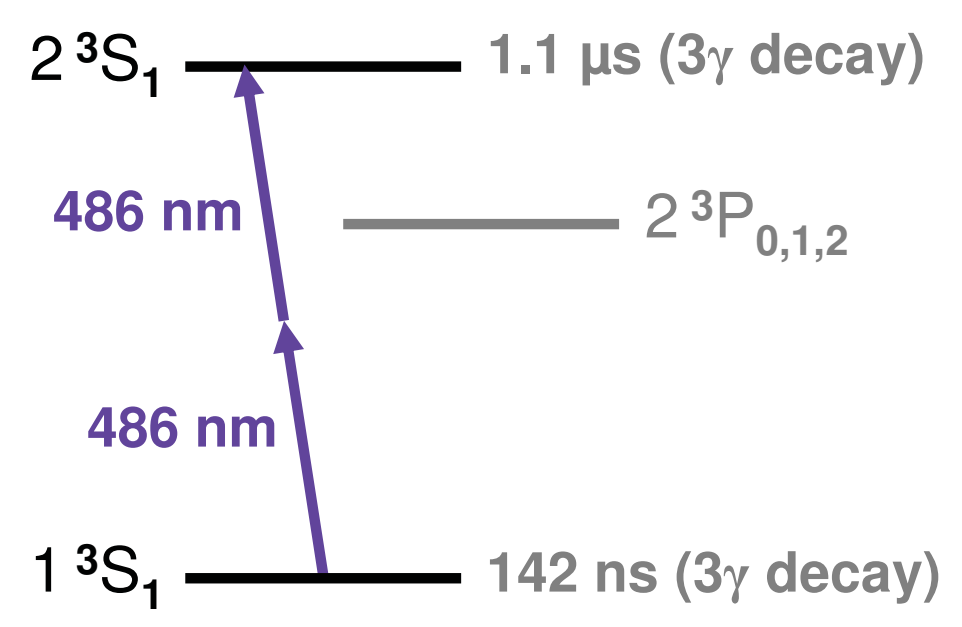

\section{The most sensitive to QED effects since they roughly scale as $1 / \mathrm{n}^{3}$}

Doppler-free two-photon spectroscopy

$\mathrm{n}=2$ population is detected through positrons counting after laser ionization

This transition was measured fort the first time with a pulsed dye laser:

$$
\text { S. Chu, A.P. Mills JR and J.L. Hall, Phys. Rev. Lett. 52, } 1689 \text { (1984) (Stanford) }
$$

and later with $\mathrm{cw}$ excitation :

$$
\text { M.S. Fee, S. Chu et al., Phys. Rev. A 48 }, 192 \text { (1993) }
$$

Result : 1233607216.4 (3.2) MHz accuracy 2.6 ppb

sufficient accuracy to test the $R_{\infty} \alpha^{4}$ QED corrections

Presently another group plans to perform a new experiment in order to improve this accuracy by a factor 5 and then check recent QED calculations P. Crivelli, C.L. Cesar and U. Gendotti, Can. J. Phys. 89, 29 (2011) 


\section{The muonium $\mu^{+}-\mathrm{e}^{-}$}

Like electrons and positrons, muons are leptons, but their mass is $\sim 207$ times larger

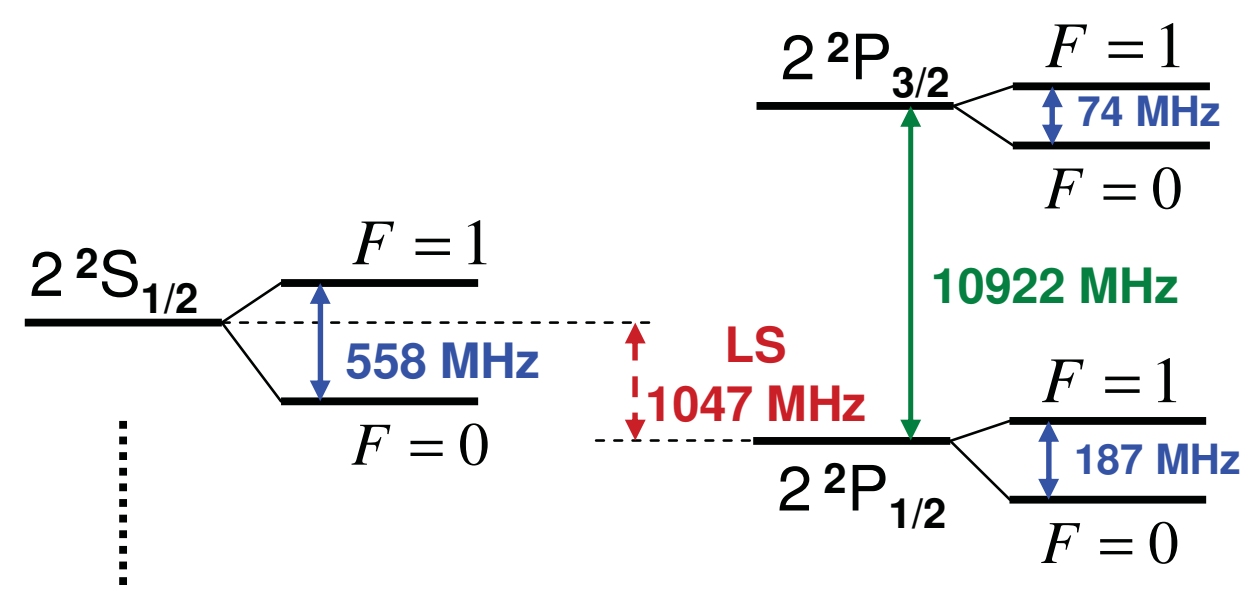

Production of muonium :

- high energy proton beam incident on a target (ex. Be)

- $\pi^{+}$are created which decay in $\mu^{+}$

- low energy muon beam incident on a gas target (ex. Ar) produces muonium atoms by electron capture

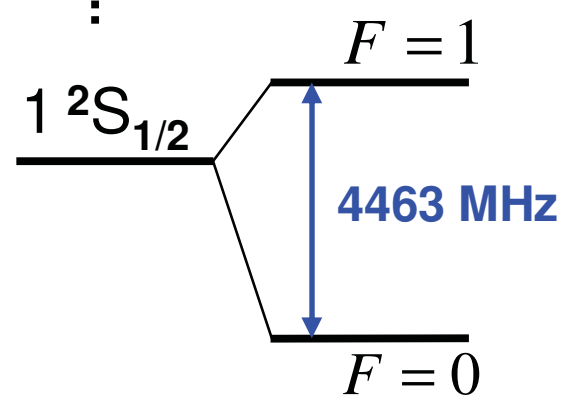

The energy levels of the muonium atom are similar to the ones of hydrogen

Ly $\alpha$ transition at $122 \mathrm{~nm}$ 


\section{RF measurement of the 2S Lamb shift in muonium}

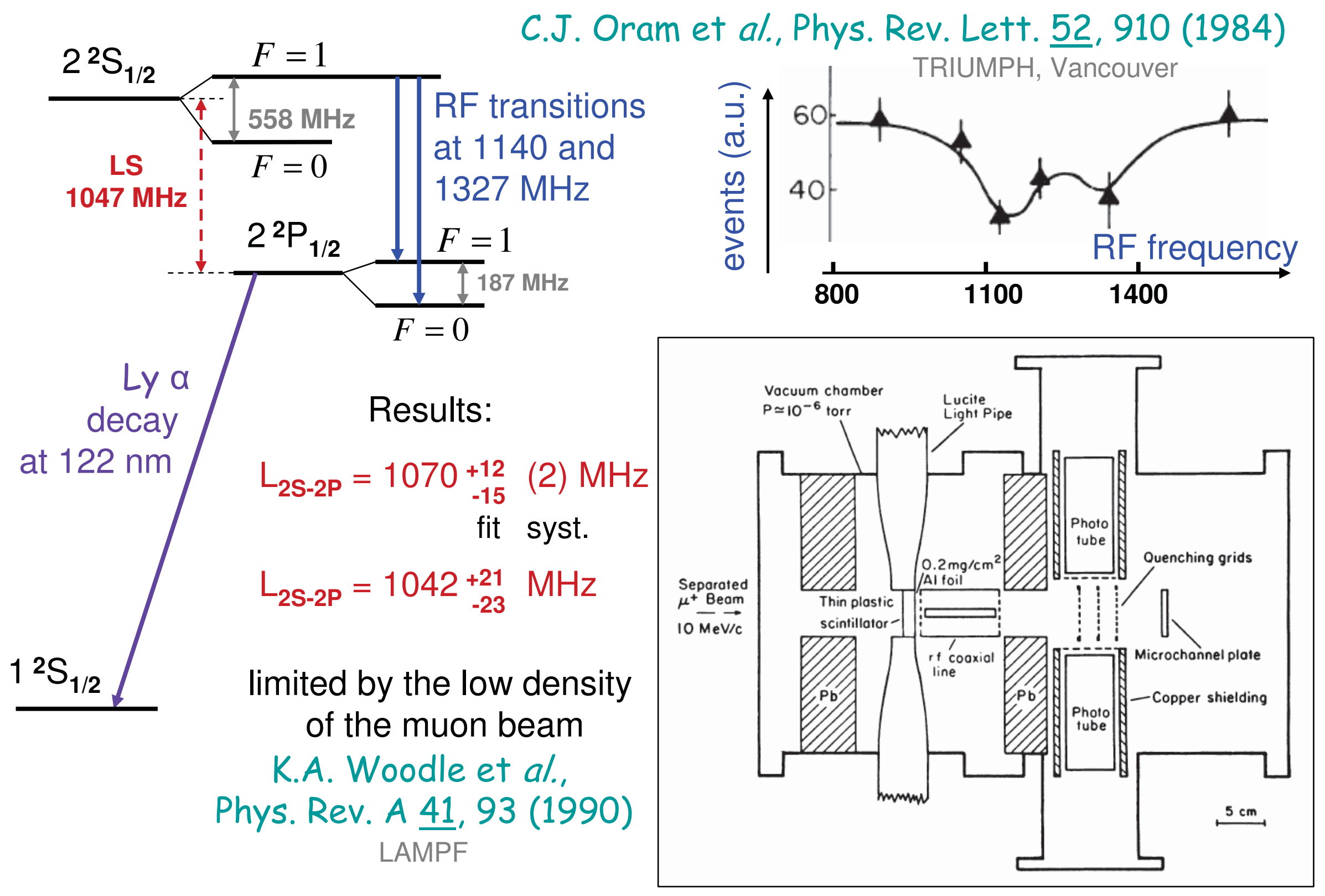




\section{Frequency measurement of the 1S-2S transition of muonium}

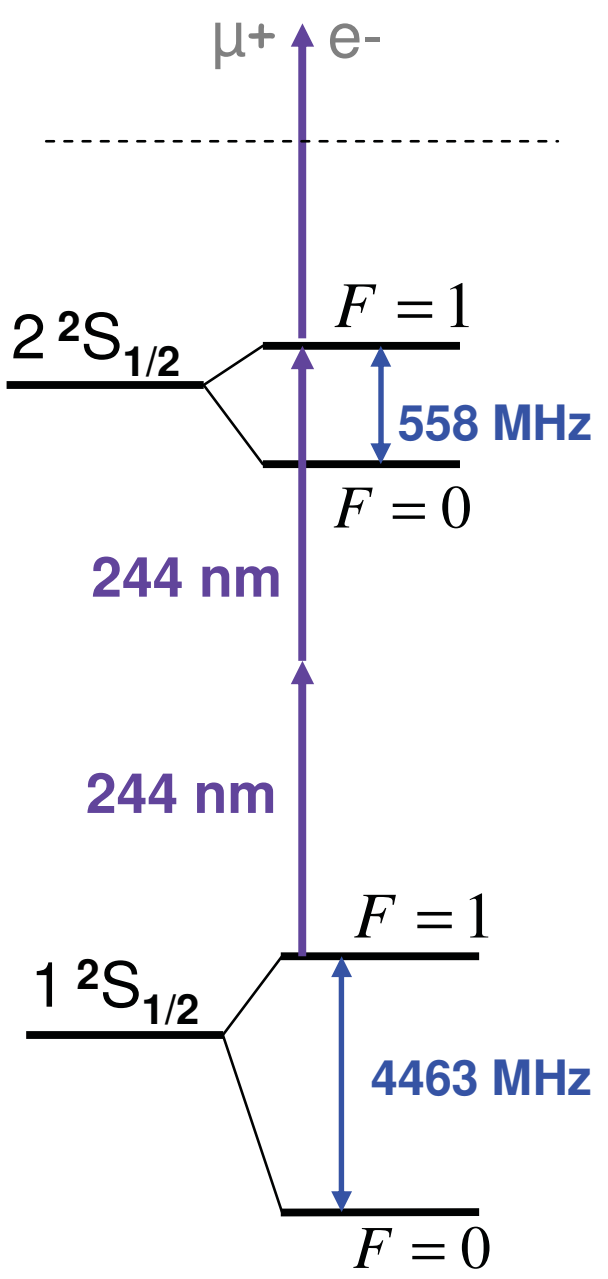

First observation of the 1S-2S Doppler-free two-photon transition with a frequency doubled dye laser

S. Chu et al., Phys. Rev. Lett. 60, 101 (1988) (Stanford) The 2S state is detected by photoionization

Frequency measurements :

F.E. Maas etal., Physics Lett. A 187, 247 (1994)

(Heidelberg, ...)

V. Meyer et al.,

Phys. Rev. Lett. 84, 1136 (2000)

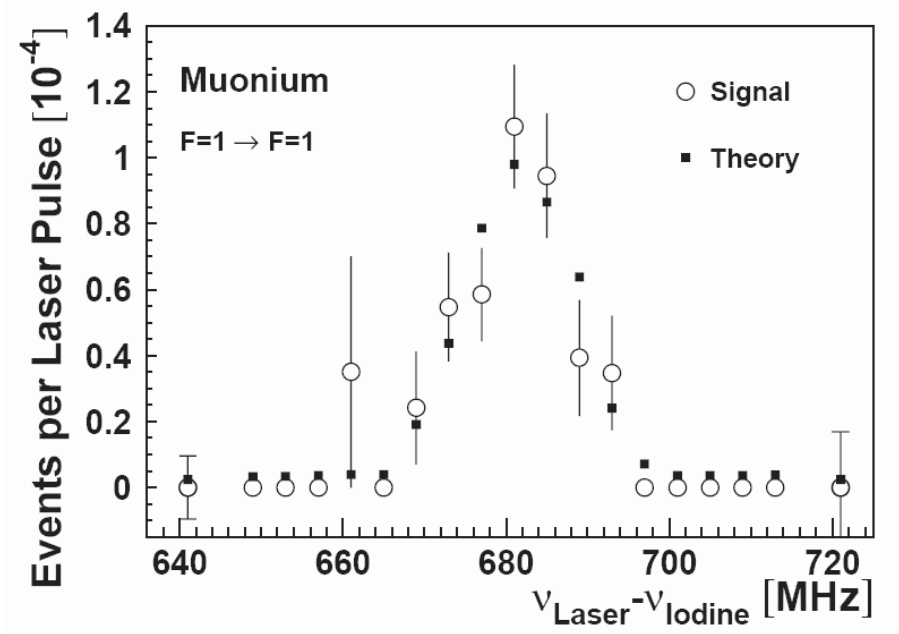

Result: $\Delta v(1 \mathrm{~S}-2 \mathrm{~S})=2455528941.0$ (9.8) MHz in good agreement with theory

The Lamb shift contribution can be deduced :

$$
\Delta v_{\mathrm{LS}}=7049.4 \text { (9.9) MHz }
$$

The exp./ theory comparaison gives the mass ratio: $\mathrm{m}_{\mu_{+}} / \mathrm{m}_{\mathrm{e}_{-}}=206.7682775$ (24) in agreement but less precise than the ratio deduced from the muon magnetic moment 


\section{The 1S hyperfine structure of muonium}

Experiments have been performed either in low or in high magnetic field

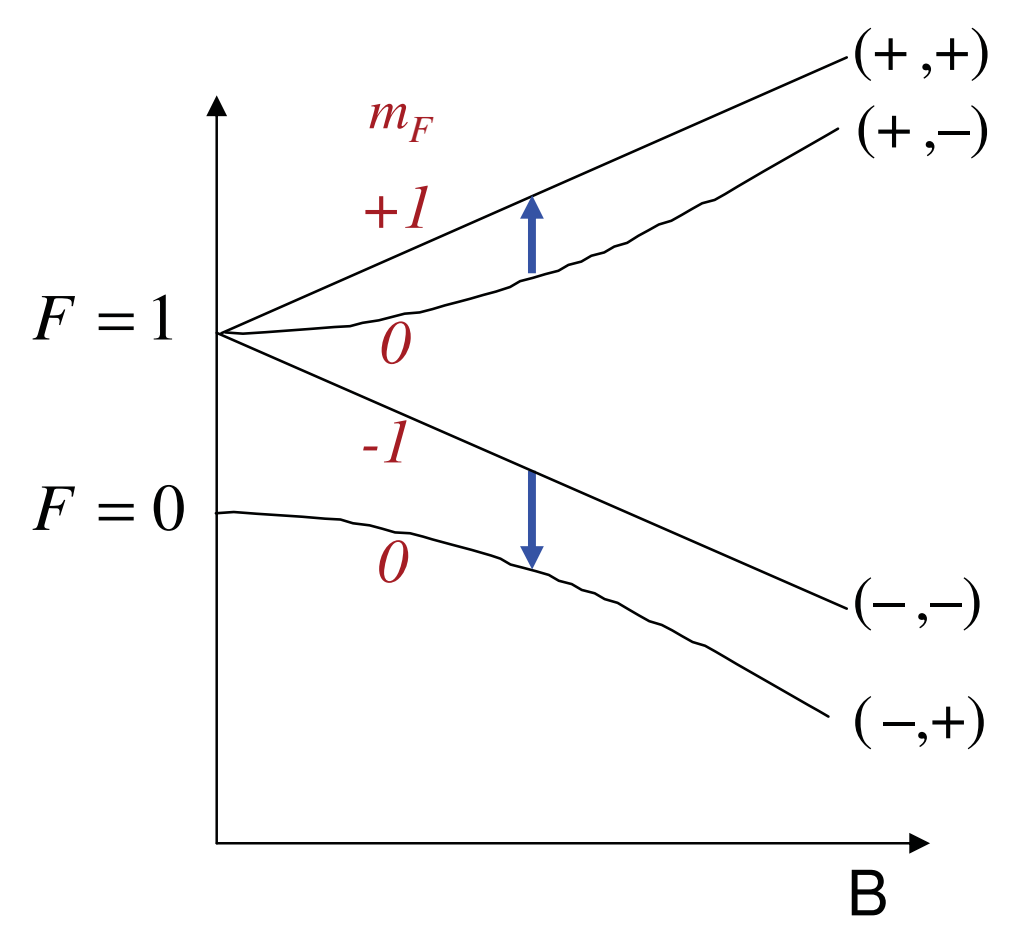

Polarized muons are captured in a microwave cavity containing Kr gas inside a superconducting magnet

Muonium is formed in states $(+,-)$ and $(-,-)$

Microwave magnetic resonance induces muon spin-flip transitions

The transition signal is detected through the angular distribution of positrons produced by the $\mu^{+}$decay

Results : $\Delta v_{\text {hfs }}=4463302765(53) \mathrm{Hz} 12 \mathrm{ppb}$

$$
\mu_{\mu_{+}} / \mu_{\mathrm{e}^{-}}=3.18334513 \text { (39) } 120 \text { ppb }
$$

Most precise results obtained in muonium

W. Liu et al., Phys. Rev. Lett. 82, 711 (1999) 


\section{Muonium / positronium : discussion}

Natural widths in muonium are limited by muon lifetime $(2.2 \mu \mathrm{s})$ since they are limited by $\mathrm{e}^{+}-\mathrm{e}^{-}$annihilation in positronium and are then shorter

Muonium is more sensible to QED corrections since its radius is smaller : vacuum polarization terms are more important Compared to positronium, QED calculations in muonium are less complicated and generally more accurate

A good agreement is found for both atoms between experimental results and theoretical predictions but tests in muonium are more stringent

In particular, the study of hyperfine structure of muonium allows to deduce values either of $\mathrm{m}_{\mu+} / \mathrm{m}_{\mathrm{e}-}$ or of the fine structure constant $\alpha$, using other experimental data and/or theoretical predictions

$$
\begin{aligned}
& m_{\mu+} / m_{e-}=206.7682670(55) 27 \text { ppb } \\
& 1 / \alpha=137.0359963(80) 58 \text { ppb }
\end{aligned}
$$

see discussion in: W. Liu et al., Phys. Rev. Lett. 82, 711 (1999) 


\section{Outline of this lecture}

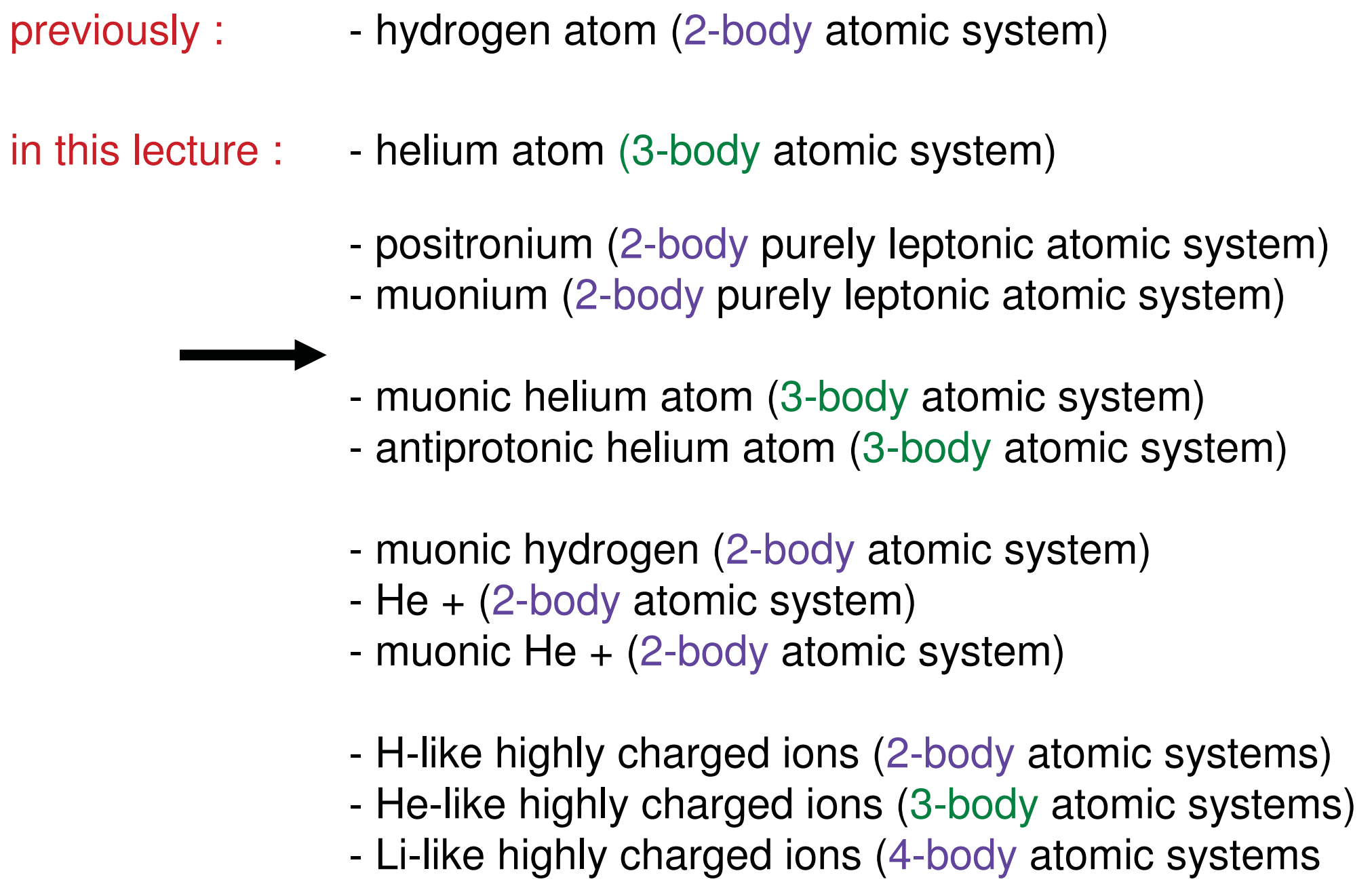




\section{The muonic helium atom}

\section{${ }^{4} \mathrm{He}^{++} \mathrm{H}^{-} \quad$ One of the two electrons of an usual He atom \\ is replaced by a negative muon}

\section{Because of their different masses,}

the radius of the $\mathrm{e}^{-}$orbit is large compared to the one of the $\mu^{-}$

This atom is similar to hydrogen with a pseudonucleus $\left({ }^{4} \mathrm{He}^{++} \mu^{-}\right)^{+}$

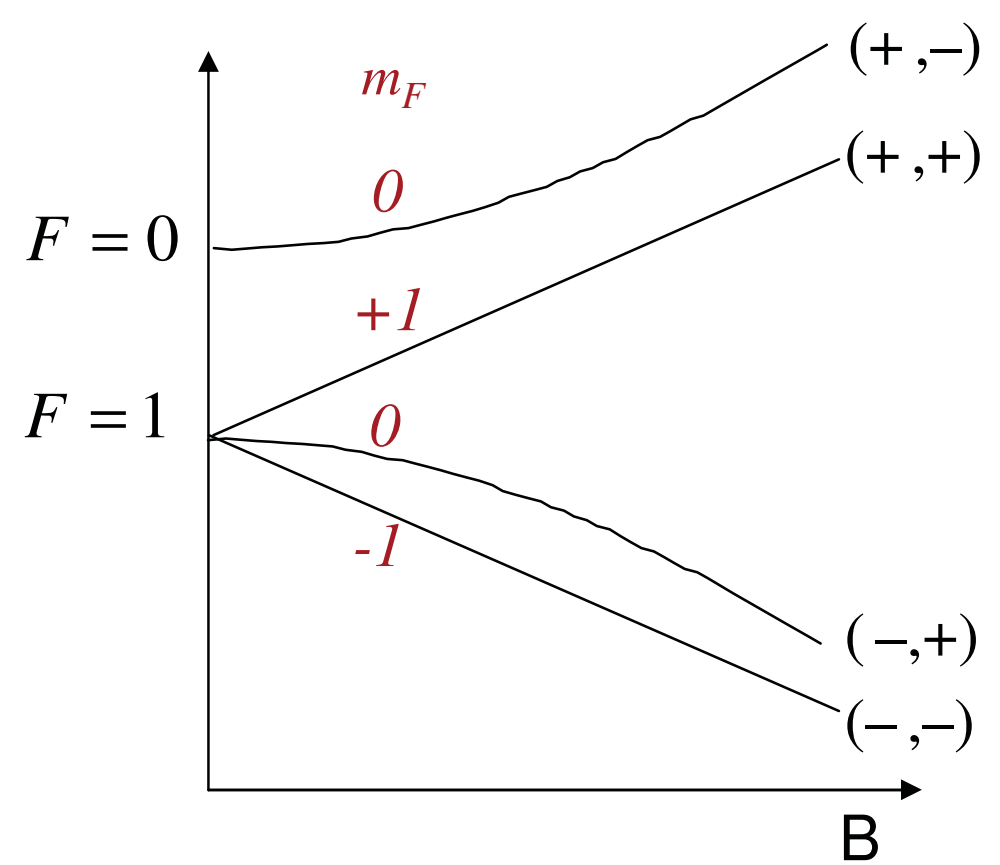

The ground state hyperfine structure

is approximately equal to the one of muonium

but inverted because $\mu^{-}$and $\mu^{+}$

magnetic moments have opposite signs

The finite size of the alpha particle has

almost negligible influence on hyperfine structure

K. Pachucki et al., Phys. Rev. A 63, 032508 (2001) 


\section{Spectroscopy of muonic helium atom}

Muonic helium atoms are formed by stopping polarized $\mu^{-}$in He gas mixed with some $\mathrm{Xe}:\left({ }^{4} \mathrm{He}^{++} \mu^{-}\right)^{+}$is formed which radiates to its ground state and then captures an electron from a Xe atom

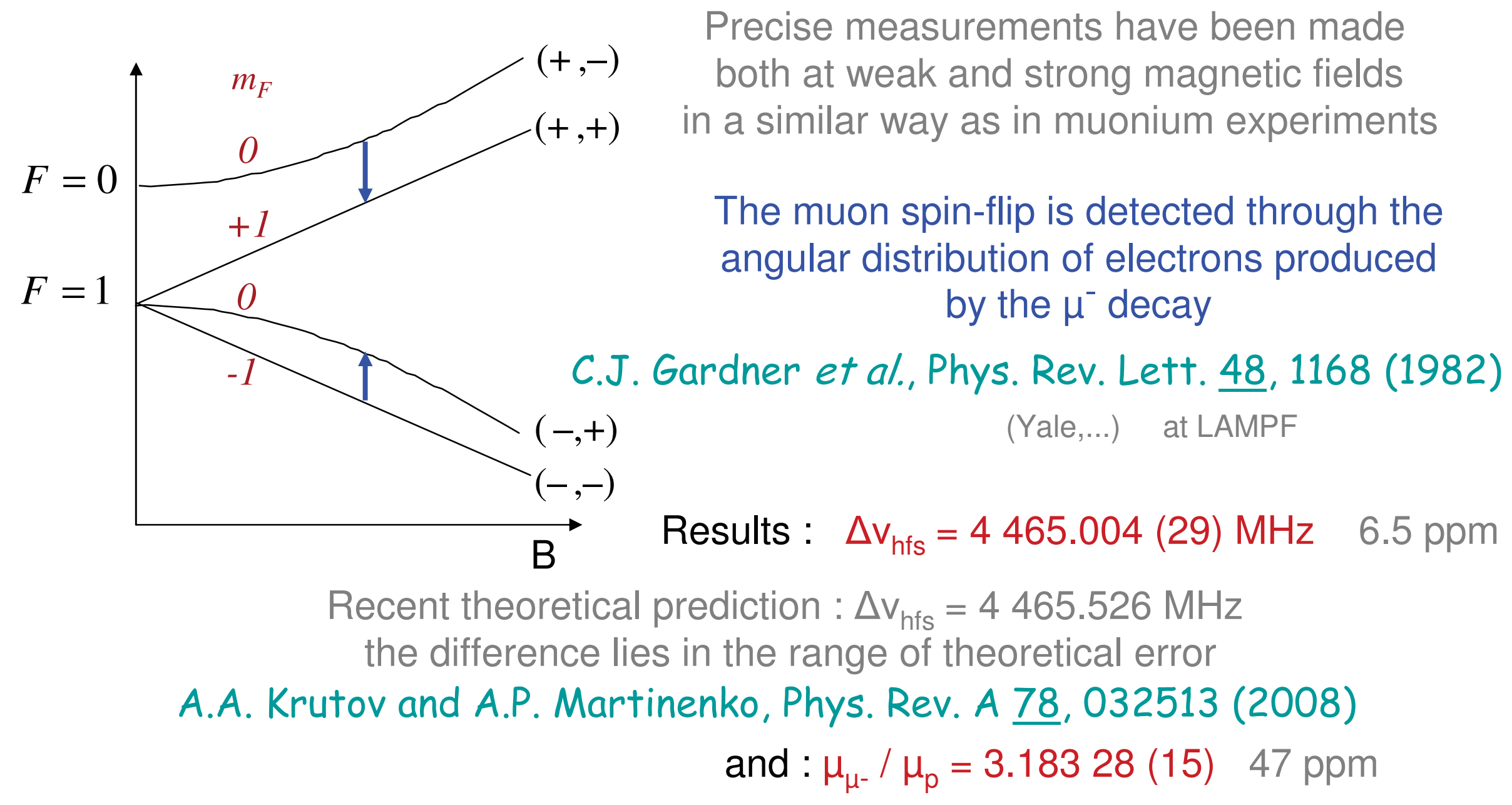




\section{Spectroscopy of antiprotonic helium}

$\overline{\mathrm{p}} \mathrm{He}^{+}$: helium atom where an electron is replaced by an antiproton

work carried out at CERN

The electron is in its ground state and the antiproton in a circular Rydberg state

$$
n \approx \ell+1 \approx 38
$$

Two-photon transitions of the type $(n, \ell) \rightarrow(n-2, \ell-2)$ by two counterpropagating UV laser beams at $139.8 \mathrm{~nm}, 193.0 \mathrm{~nm}$, and $197.0 \mathrm{~nm}$

The comparison of the three measured frequencies with QED calculations allows to derive the antiproton-to-electron mass ratio $1836.1526736(23)$ in agreement with the proton-to-electron value

$$
\text { M. Hori et al., Nature } \underline{475}, 484 \text { (2011) }
$$

The study of hyperfine structure in antiprotonic helium leads also to the determination to the antiproton magnetic moment in agreement with then one of the proton within $2.9 \times 10^{-3}$

T. Pask et al., Physics Letters B 678, 55 (2009) 


\section{Muonic hydrogen and the proton charge radius puzzle}

Exotic particles can been used:

- to eliminate proton size effects encountered in hydrogen atom

- but also to attempt to determine the proton size

We have seen that the measured Lamb shifts in hydrogen need the knowledge of the proton radius to be used to test QED predictions

The proton has a structure :

2 quarks up $(2 / 3$ e $)+1$ quark down $(-1 / 3$ e)

+ strong interaction (gluons)

The proton charge radius is defined as:

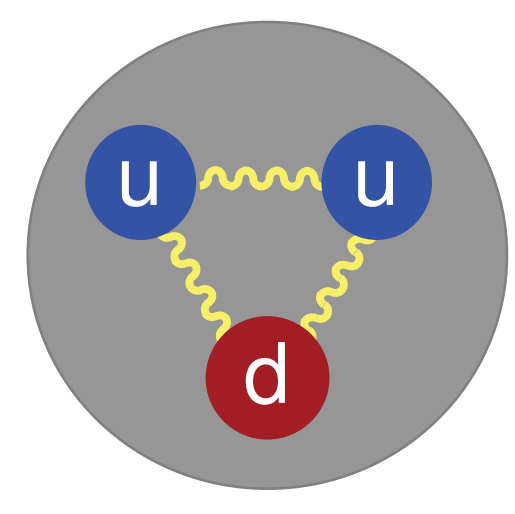

$$
\begin{aligned}
& r_{\mathrm{p}}=\sqrt{\left\langle r^{2}\right\rangle} \quad \text { where }\left\langle r^{2}\right\rangle=\frac{\int r^{2} \rho(r) \mathrm{d}^{3} r}{\int \rho(r) \mathrm{d}^{3} r} \quad \begin{array}{l}
\text { and } \rho(r) \text { is the } \\
\text { proton charge distribution }
\end{array} \\
& r_{\mathrm{p}} \sim 0.8 \mathrm{fm}
\end{aligned}
$$




\section{Determinations of the proton charge radius}

- From spectroscopic measurements

ex. : in hydrogen atom (e-p)

$E(n, l, j)=\underbrace{\text { Dirac }+ \text { recoil }}_{\text {exact }}+L(n, l, j)$

The Lamb shift $L(n, l, j)$ includes :

- QED radiative corrections $\left(1 / \mathrm{n}^{3}\right)$

- relativistic recoil

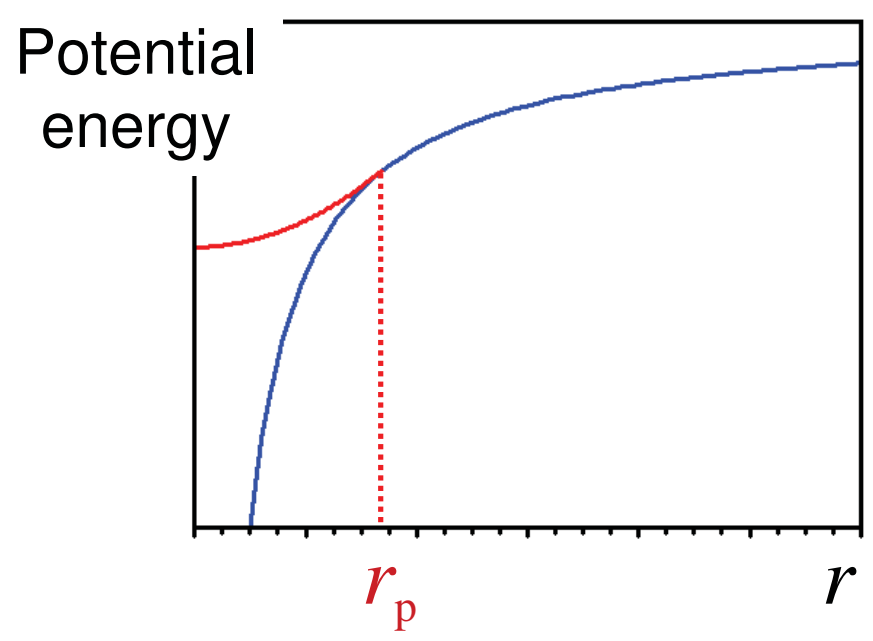

- nuclear size effect $\left(1 / n^{3}\right)$

$f(1 S-2 S)=2466061413187035(10) \mathrm{Hz}$

- From electron - proton scattering

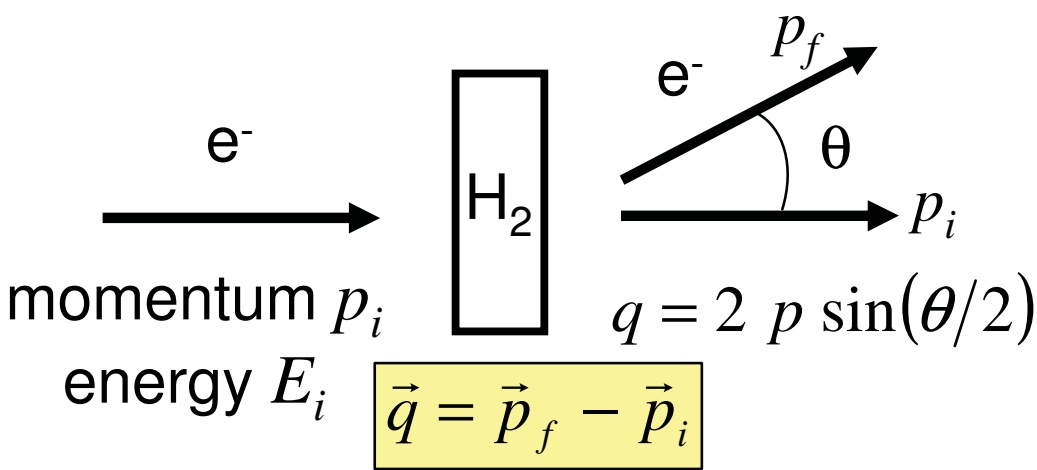

J.C. Bernauer, Can. J. Phys. 8 5, 419 (2007)

$$
\begin{aligned}
& \frac{d \sigma}{d \Omega}\left(E_{i}, \theta\right) \approx \underbrace{\left[\frac{a^{2}}{\sin ^{4}(\theta / 2)}\right]}_{\text {Rutherford }} G\left(q^{2}\right) \\
& G\left(q^{2}\right)=\int d^{3} r e^{i q r} \frac{\rho(r)}{4 \pi} \approx 1-\frac{r_{p}{ }^{2}}{6} q^{2} \\
& r_{\mathrm{p}} \text { is deduced from an extrapolation } \\
& \text { to } q \rightarrow 0
\end{aligned}
$$




\section{The muonic hydrogen Lamb shift}

It is much more sensitive to the proton radius than the hydrogen Lamb shift since the Bohr radius is only $a_{0} / 207$

The various contributions to the 2S-2P Lamb shift :

relative

\begin{tabular}{|c|c|c|c|c|}
\hline $2 \mathrm{~S}-2 \mathrm{P}$ & self-energy & vacuum pol. & $r_{\mathrm{p}}$ & total \\
\hline $\mathrm{e}-\mathrm{p}$ & $1085.8 \mathrm{MHz}$ & $-26.9 \mathrm{MHz}$ & $0.146 \mathrm{MHz}$ & $1057.8 \mathrm{MHz}$ \\
\hline$\mu-\mathrm{p}$ & $0.1 \mathrm{THz}$ & $-49.94 \mathrm{THz}$ & $0.93 \mathrm{THz}$ & $-49.05 \mathrm{THz}$ \\
\hline
\end{tabular}

contribution

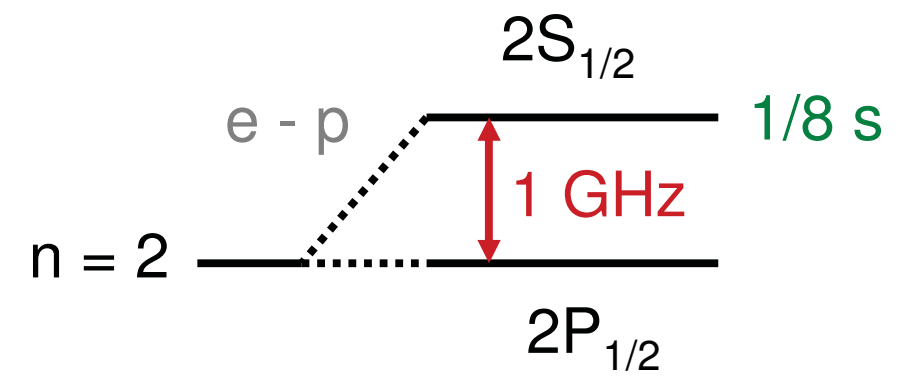

oscillator strengths ratio : $\left(f_{\mu-p} / f_{e-p}\right) \propto 1 /(207)^{3} \approx 10^{-7}$

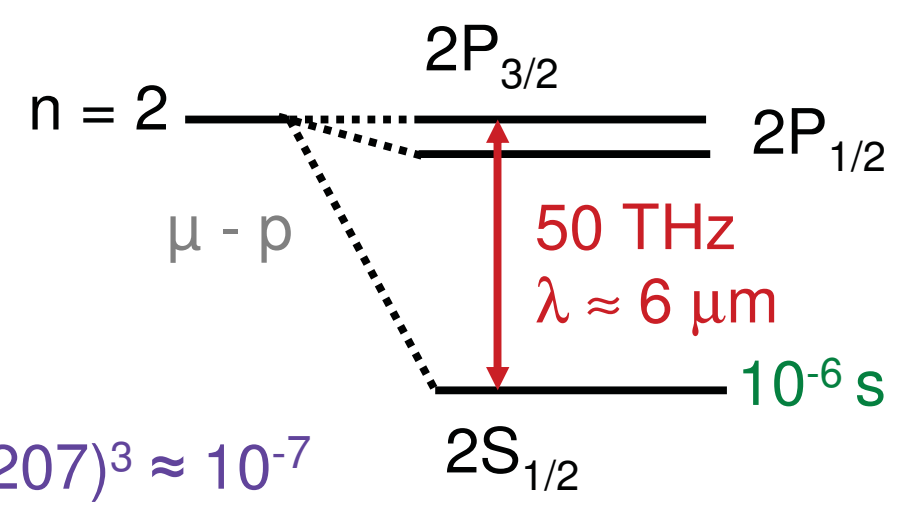

$$
\begin{array}{r}
\Delta \mathrm{E}_{2 \mathrm{~S}(\mathrm{~F}=1)-2 \mathrm{P}(\mathrm{F}=2)}=50.7702(12)-1.2634 r_{\mathrm{p}}{ }^{2}-0.0084 r_{\mathrm{p}}{ }^{3}=49.8063(149) \mathrm{THz} \\
\text { with } r_{\mathrm{p}}=0.8760(68) \mathrm{fm}
\end{array}
$$




\section{Production of muonic hydrogen}

\section{CREMA \\ collaboration}
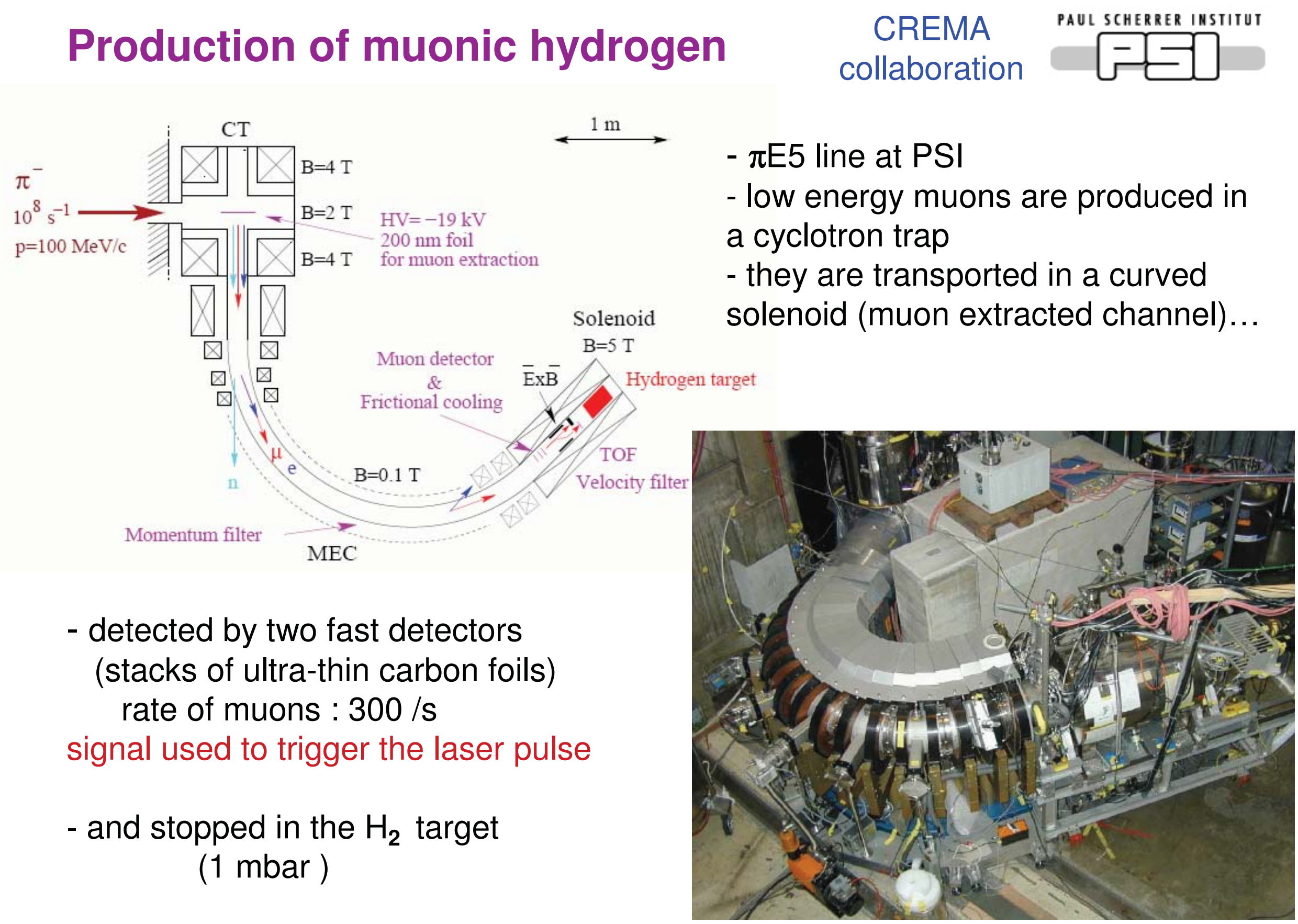


\section{Production of muonic hydrogen in the $2 \mathrm{~S}$ metastable state ...}

- the muon is captured in a highly excited state which decays at $99 \%$ to the ground state emitting a « prompt » $\mathrm{X}$ ray $(\mathrm{Ka}, \mathrm{K} \beta, \ldots)$

- $X$ rays are detected with LAAPDs (large area avalanche photodiodes) placed above and below the muon stop volume

$-1 \%$ of the stopped muons decay to the long-lived $2 \mathrm{~S}$ state

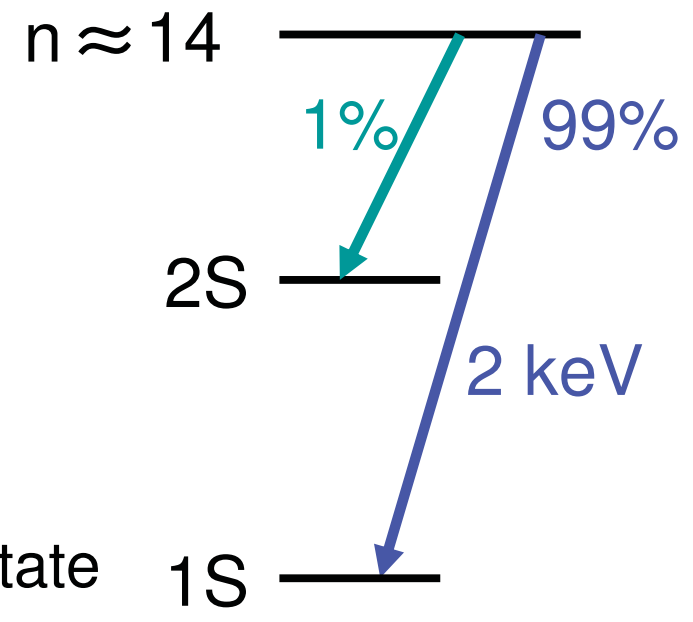

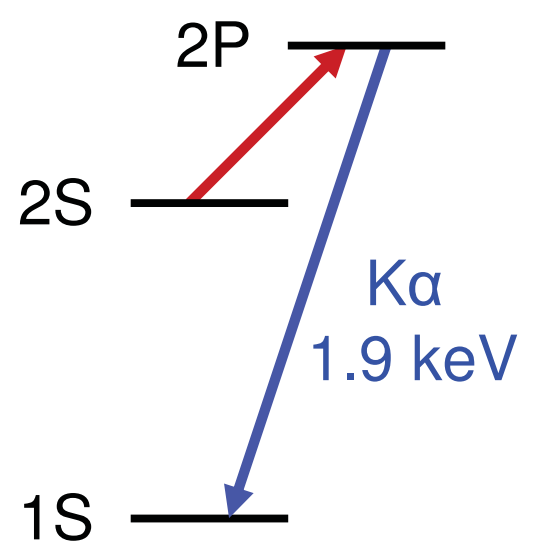

The signature of the signal is the detection of $\mathrm{Ka}$, in time coincidence with the laser excitation, and of the electron originating from the muon decay (muon lifetime is $2.2 \mu \mathrm{s}$ )

\section{... and excitation of the transition}

A short laser pulse at $6 \mu \mathrm{m}$ drives the 2S-2P transition - the transition is detected through the $1.9 \mathrm{keV}$ Ka decay of the $2 \mathrm{P}$ state (« delayed $\mathrm{X}$ ray)

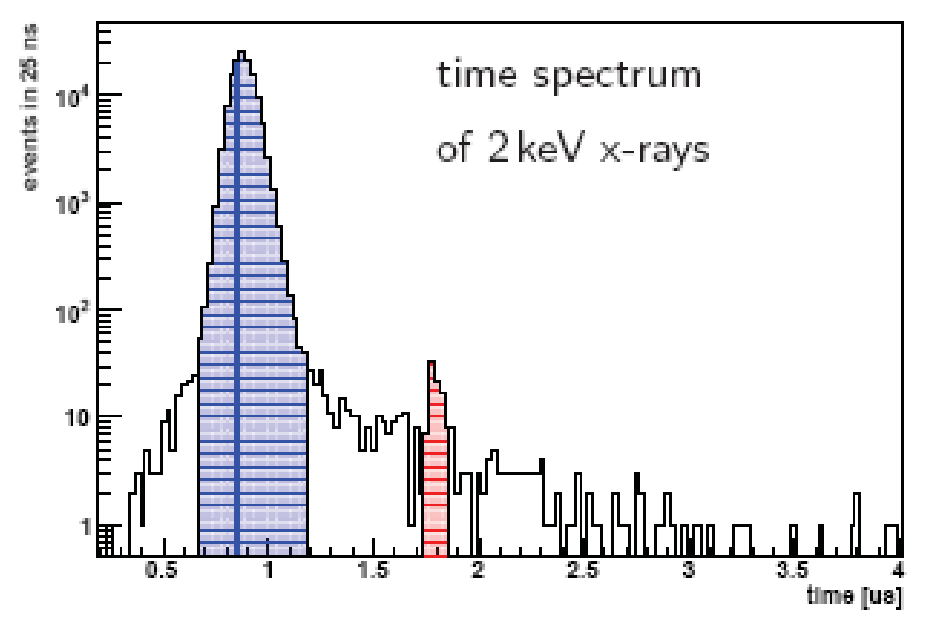




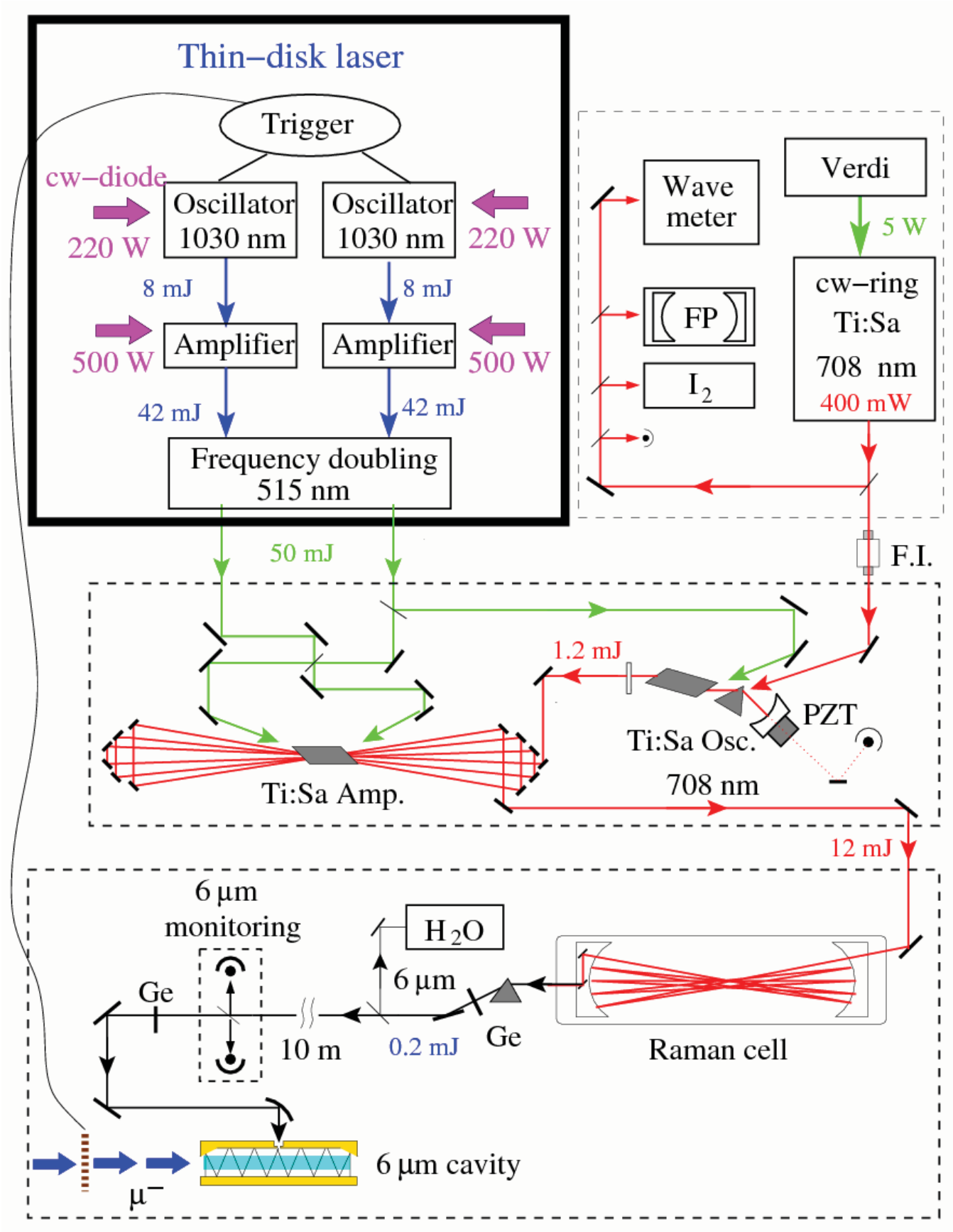

\section{The laser chain}

to produce a $6 \mu \mathrm{m}$ tunable laser pulse

- thin disk laser (1030 nm)

$\mu^{-}$triggered

+ LBO (515 nm)

- pulsed TiSa oscillator + amplifier (cw TiSa seeded at $708 \mathrm{~nm}$ )

- Raman cell for frequency conversion

1st Stokes

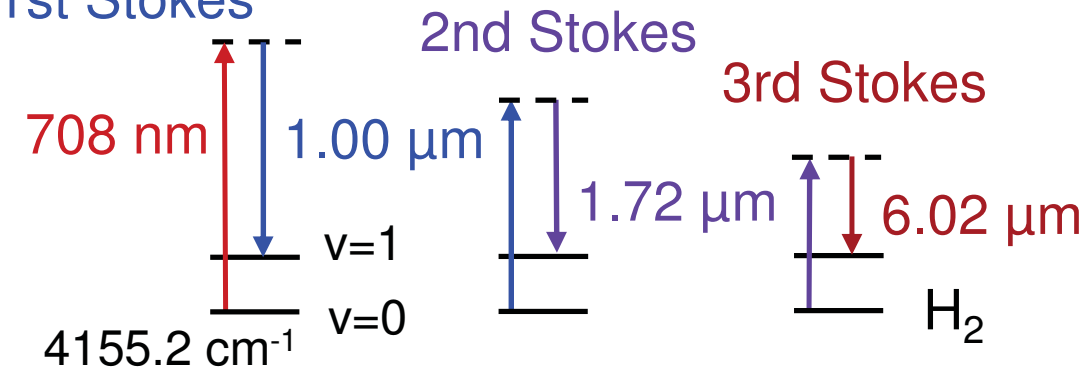

- Multipass cavity at $6 \mu \mathrm{m}$ surrounding the $\mathrm{H}_{2}$ target 
$2 S_{1 / 2}(F=1)-2 P_{3 / 2}(F=2)$ transition observed in muonic hydrogen in 2009

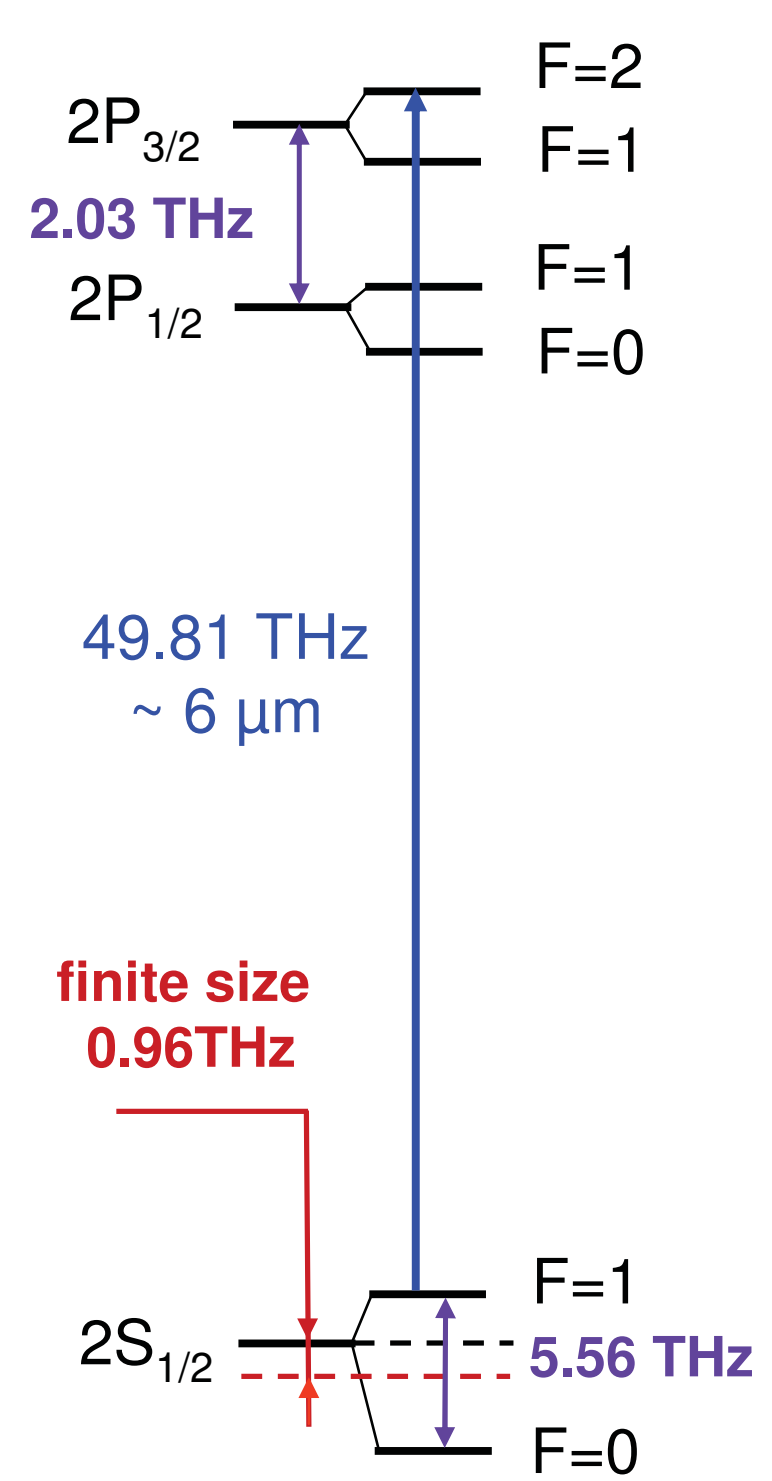

- 550 events measured

155 backgrounds

- 31 FP fringes

- 250 hours

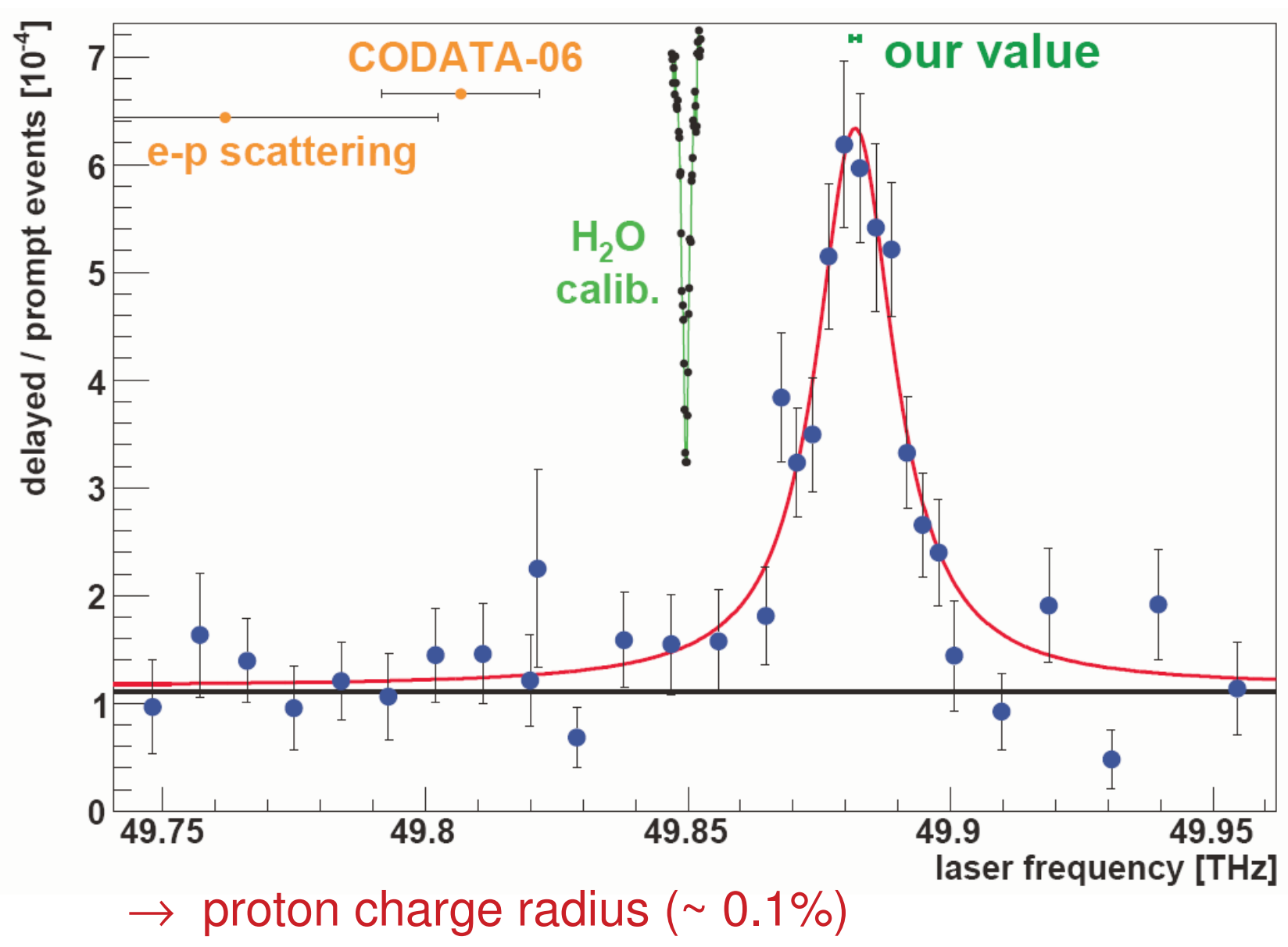


Result and comparison with other best determinations

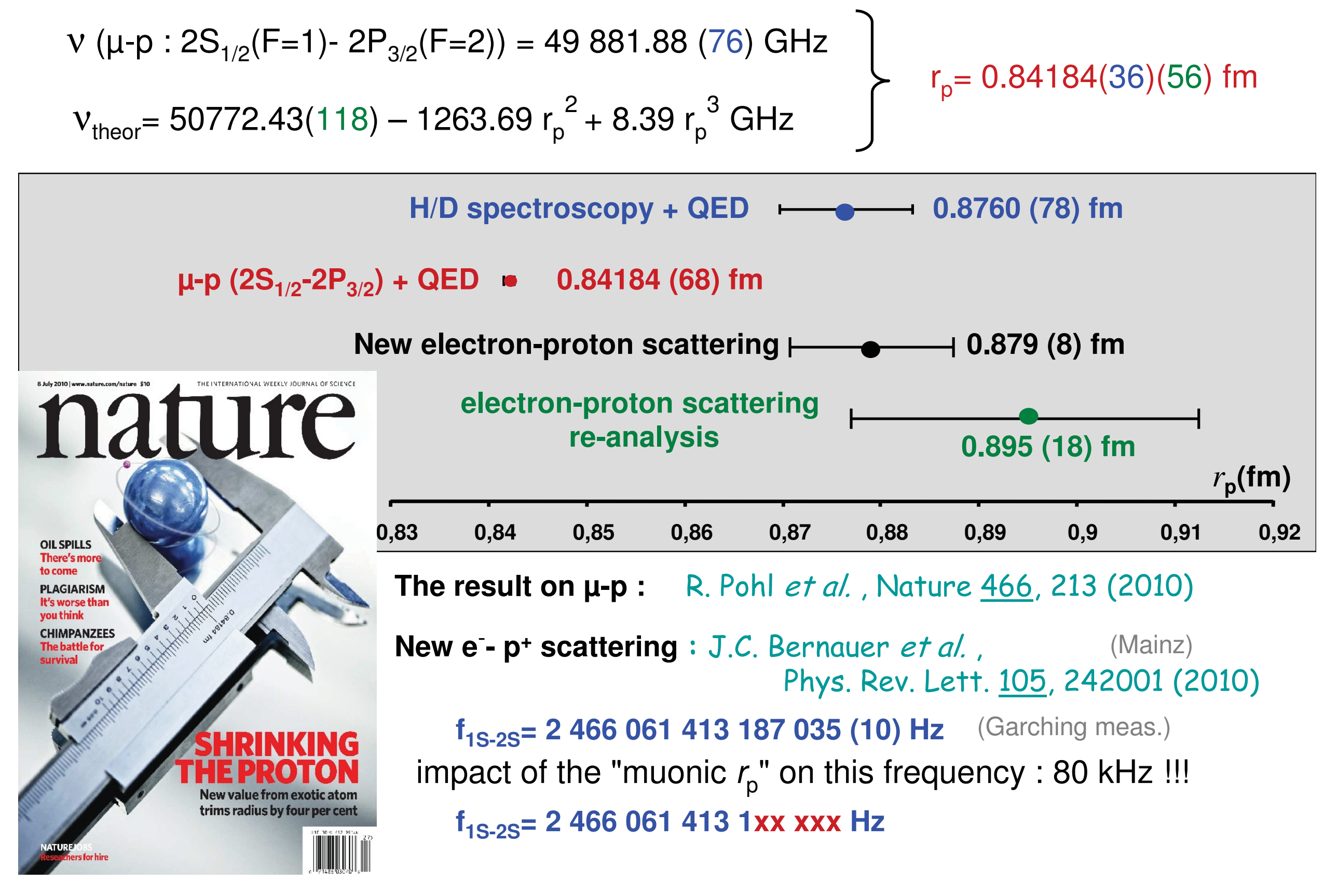




\section{Other transitions observed at PSI}

- in $\mu$-p atom : $\quad 2 S_{1 / 2}(F=0)-2 P_{3 / 2}(F=1) \quad$ at $5.5 \mu m$

The hyperfine structure will also give the Zeemach radius of the proton

- in $\mu$-d atom : $\quad 2 \mathrm{~S}_{1 / 2}(\mathrm{~F}=3 / 2)-2 \mathrm{P}_{3 / 2}(\mathrm{~F}=5 / 2) \quad$ at $5.9 \mu \mathrm{m}$

$$
\begin{array}{r}
2 S_{1 / 2}(F=1 / 2)-2 P_{3 / 2}(F=3 / 2) \\
\text { and } 2 S_{1 / 2}(F=1 / 2)-2 P_{3 / 2}(F=1 / 2) \quad \text { at } 5.7 \mu \mathrm{m}
\end{array}
$$

Analysis is in progress ...

\section{Conclusion}

- Since 2010, QED calculations have been checked in e-p and $\mu-p$ but the results obtained in the two systems remain unconsistent

- It cannot be explained by the proton structure

R.J. Hill and G. Paz, Phys. Rev. Lett. 107, 160402 (2011)

- Is it the signature of "new physics" ?

V. Barger et al., Phys. Rev. Lett. 106, 153001 (2011)

Spectroscopic measurements of various lines in hydrogen and in other simple atomic systems must be pursued 


\section{On going experiments in hydrogen atom ...}

- 1S-2S hydrogen spectroscopy in MPQ Garching hydrogen cooled with magnetic field

-1S-3S hydrogen picosecond spectroscopy in MPQ Garching

-1S-3S (and 1S-4S) hydrogen cw spectroscopy in LKB Paris

Frequency mixing in place of frequency doubling to efficiently produce $205 \mathrm{~nm}$ radiation (in progress)

- Hydrogen 2S-nS/nD spectroscopy at NPL

Improved version of the previous Paris experiment : $n>4$, frequency comb, optical production of metastable atoms

J.L. Flowers et al., IEEE Trans. Instrum. Meas. 56, 331 (2007) 


\section{... and in $\mathrm{He}^{+}$}

As for hydrogen, the charge rms radius of its nucleus (the alpha-particle ${ }^{4} \mathrm{He}^{++}$) is derived from scattering data : $r=1.681(4) \mathrm{fm}$

$$
\text { I. Sick, Phys. Rev. C 77, 041302(R) (2008) }
$$

and the Lamb shift determination in this $\mathrm{H}$-like ion provides a test of QED calculations

Scaling factors in $\mathrm{H}$-like ions

- Energy $Z^{2}$

- Dipole $\quad \sim Z^{-1}$

- 1 photon decay $\quad \sim Z^{4}$

- 2 photon decay $\quad \sim \mathrm{Z}^{6} \rightarrow$ 2S lifetimes : in $\mathrm{H}: 0.14 \mathrm{~s}$

- 1 photon excitation $\sim Z^{-6}$

in $\mathrm{He}^{+}: 1.9 \mathrm{~ms}$

- 2 photon excitation $\quad \sim Z^{-12} \rightarrow$ very low probalility for the excitation of the1S-2S transition!

M. Haas et al., Phys. Rev. A 73, 052501 (2006) 


\section{Prospects in hydrogen-like $\mathrm{He}^{+}$ions}

-1S-2S He ${ }^{+}$femtosecond spectroscopy at MPQ Garching

1S-2S two-photon transition at $64 \mathrm{~nm}$ excited in trapped ions with a frequency comb

$$
\text { M. Herrmann et al., Phys. Rev. A 79, } 052505 \text { (2009) }
$$

- 2S-2P Lamb shift measurement in muonic helium ion $\left(\mathrm{He}^{++} \mu^{-}\right)^{+}$

\section{Planned in 2013 at PSI by the CREMA collaboration}

The transition is in the $800-960 \mathrm{~nm}$ range

The contribution of the finite size effect to the 2S-2P Lamb shift is $\sim 20 \%$

The frequency measurement will lead to a determination of the charge radius (limited by the polarisability contribution) to be compared to scattering value $\rightarrow$ solve the "proton puzzle" ?

$$
\text { A Antognini et al., Can. J. Phys. 89, } 47 \text { (2011) }
$$

These both measurements in $\mathrm{He}$ and muonic helium ions will help to clarify the present discrepancy in hydrogen and to test bound states QED at a more sensitive level (two-loop effects, $B_{60}$ and $B_{71}$ terms) 


\section{Measurement of the 2S Lamb shift in $\mathrm{He}^{+}$}

by the anisotropy method, as already discussed in $\mathrm{H}$ atom

The two-loop correction to the self energy of the electron scales as $Z^{5}$ contribution : $-1.339 \mathrm{MHz}$ in $\mathrm{He}^{+}$compared to $-0.0418 \mathrm{MHz}$ in $\mathrm{H}$

A fast beam of $\mathrm{He}^{+}(2 \mathrm{~S})$ atoms is subjected to a static electric field The total Lyman a fluorescence is detected in two orthogonal directions

The measured anisotropy $R=\left(\mathrm{I}_{\|}-\mathrm{I}_{\perp}\right) /\left(\mathrm{I}_{\|}+\mathrm{I}_{\perp}\right)$ is proportional to the Lamb shift

The measured Lamb shift is 14041. 13(17) MHz

A. van Winjngaarden, J. Kwela and G.W.F. Drake, Phys. Rev. A 43, 3325 (1991)

A. van Winjngaarden, F. Holuj and G.W. Drake, Phys. Rev. A 63, 012505 (2000)

Excellent agreement with the theoretical value 14041.18(13) $\mathrm{MHz}$ 


\section{Outline of this lecture}

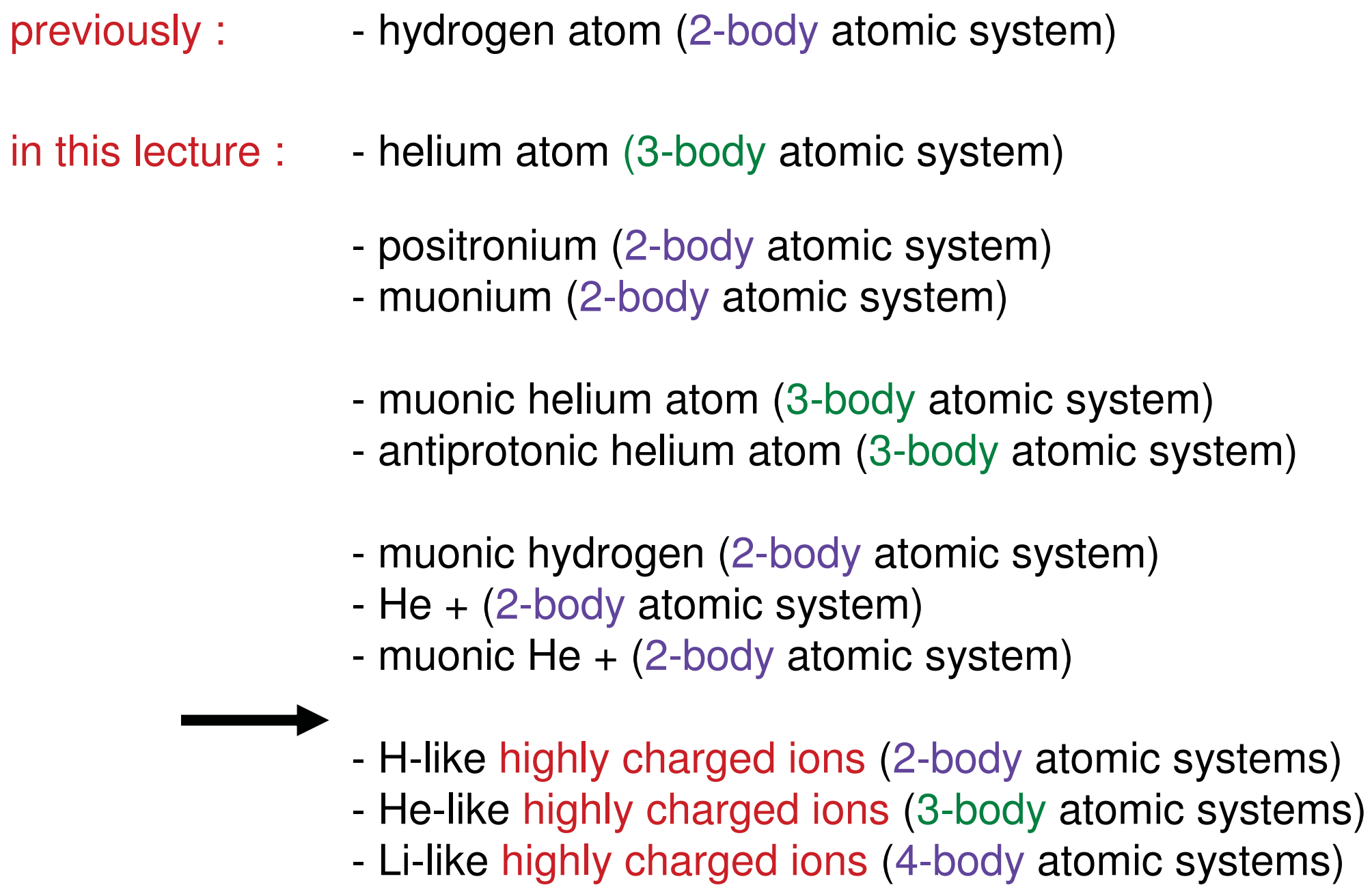




\section{Test QED with highly charged ions ( $\mathrm{HCl})$}

Other hydrogen-like atomic systems with $Z>>1$

$\left(\mathrm{Z} \alpha \sim 10^{-2} \rightarrow \mathrm{Z} \alpha \sim 1\right)$

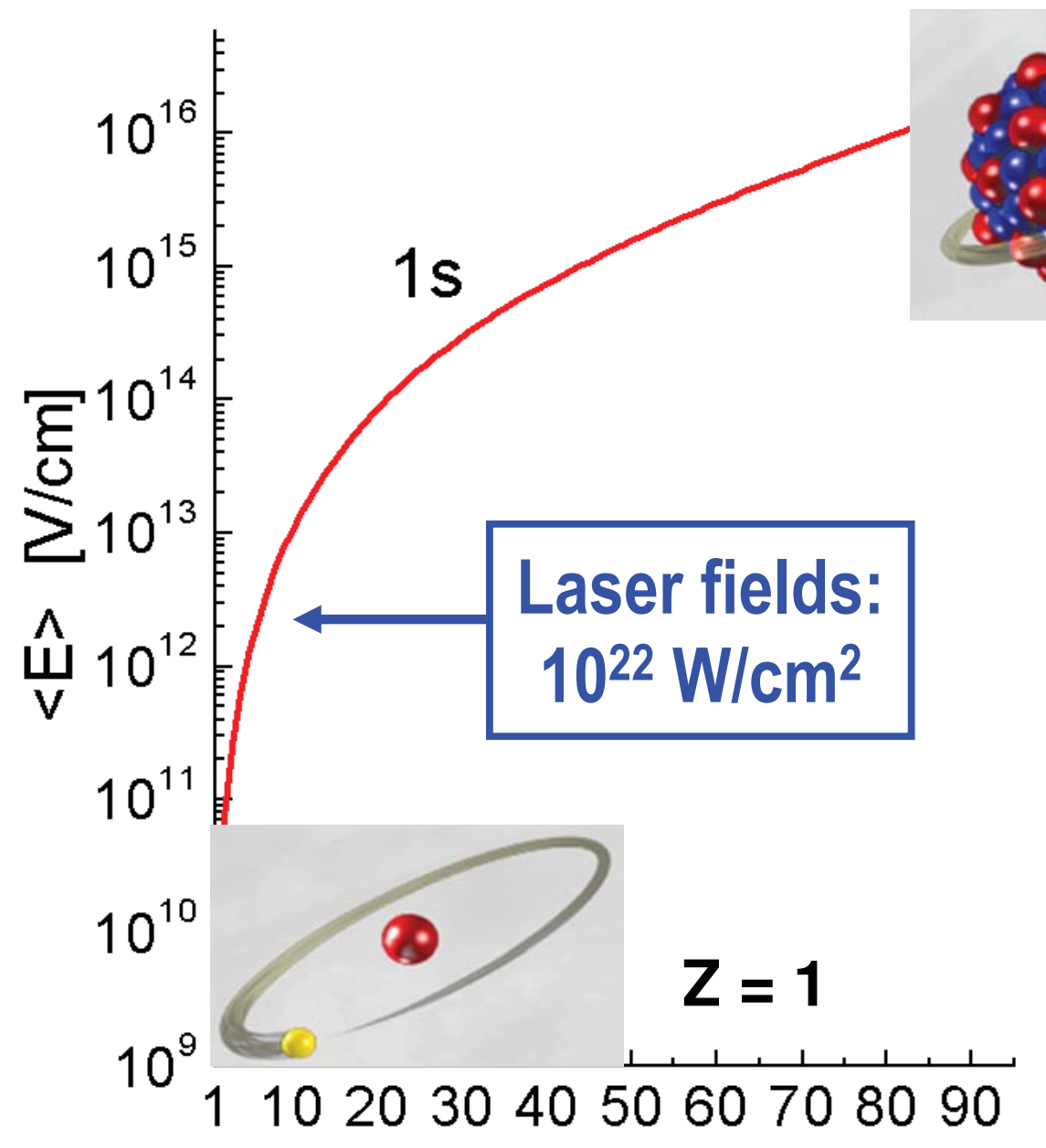

Nuclear Charge, $Z$

$$
E_{n}=m c^{2} \frac{(Z \alpha)^{2}}{2 n^{2}}
$$

An electron in an extremely strong Coulomb field

$Z=92$

$$
r_{0}=\frac{\hbar}{Z \alpha m c}
$$

Increased :

- relativistic corrections

- QED effects

- nuclear structure effects

in $\mathrm{HCl}$ series expansion in $\mathrm{Za}$ is not appropriate 


\section{S Lamb shift in $\mathrm{H}$-like highly charged ions}

The binding energy varies as $Z^{2}$ and QED corrections as $Z^{4}$

$\bullet \mathrm{Z}=1$ : hydrogen $(-13.6 \mathrm{eV})$

$\begin{array}{lll}\text { Lamb shift } & 3.5 \times 10^{-5} \mathrm{eV} & \mathrm{RF} \\ \text { Vacuum polarization } & 2.5 \% & \\ \text { Nuclear size } & 0.01 \%\end{array}$

- $Z$ = $14:$ H-like silicon (-2.6 keV)

Lamb shift

Vacuum polarization

Nuclear size

$0.48 \mathrm{eV}$

optical

$5.9 \%$

$0.5 \%$

- $\mathrm{Z}=92: \mathrm{H}$-like uranium $(-177 \mathrm{keV})$

Lamb shift

$462 \mathrm{eV}$

X-Rays

Vacuum polarization

$13.8 \%$

Nuclear size

$30.4 \%$

Frequency domain 


\section{Production, storage and cooling of $\mathrm{HCl}$}

\section{Electron Beam Ion Trap}
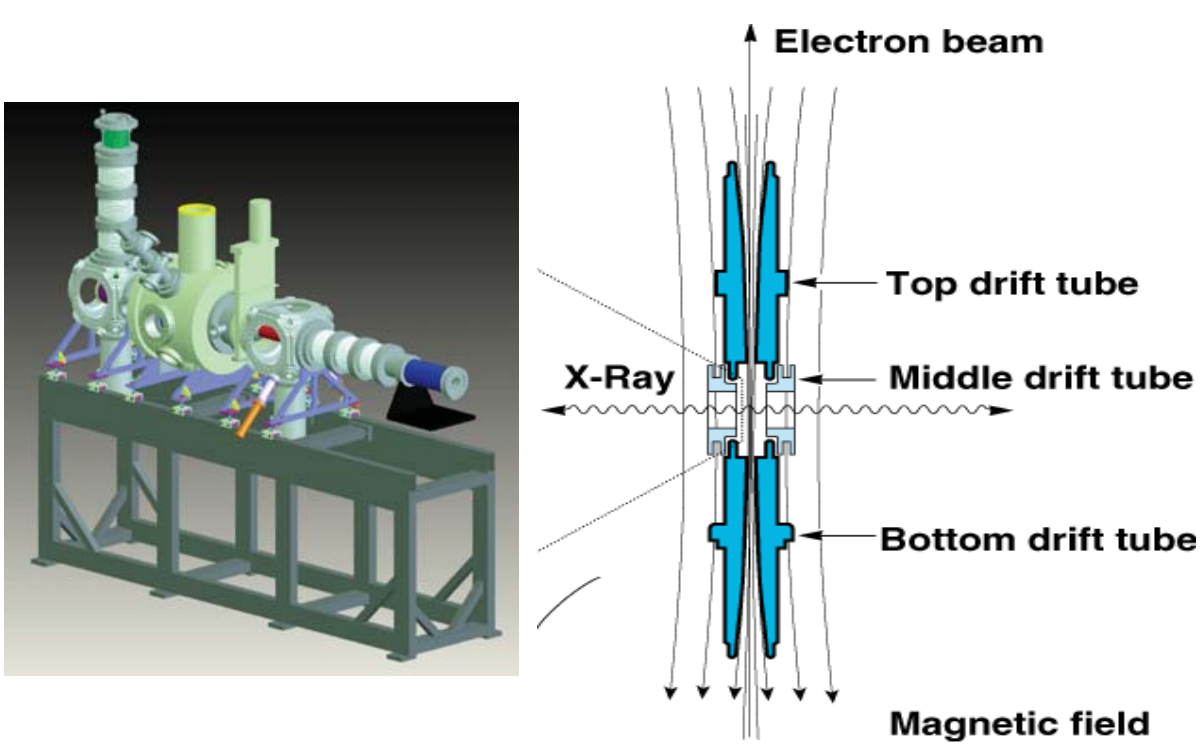

Cooling in traps

resistive cooling evaporative cooling laser cooling electron cooling

\section{Storage Ring}

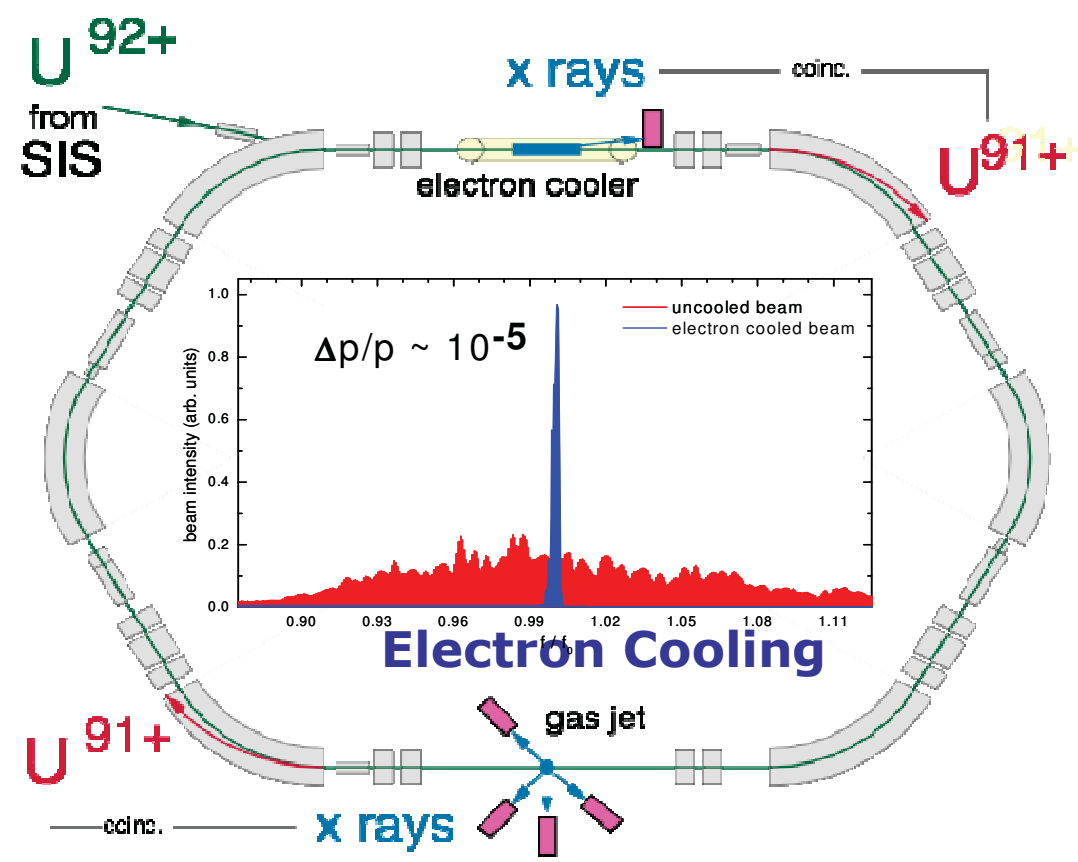

Cooling in Storage Rings electron cooling stochastic cooling laser cooling

Highly-charged ions up to bare, $\mathrm{H}$-like and He-like uranium can be produced 


\section{S Lamb shift spectroscopy in H-like uranium}

Measurement performed at the ESR storage ring in GSI (Darmstadt)
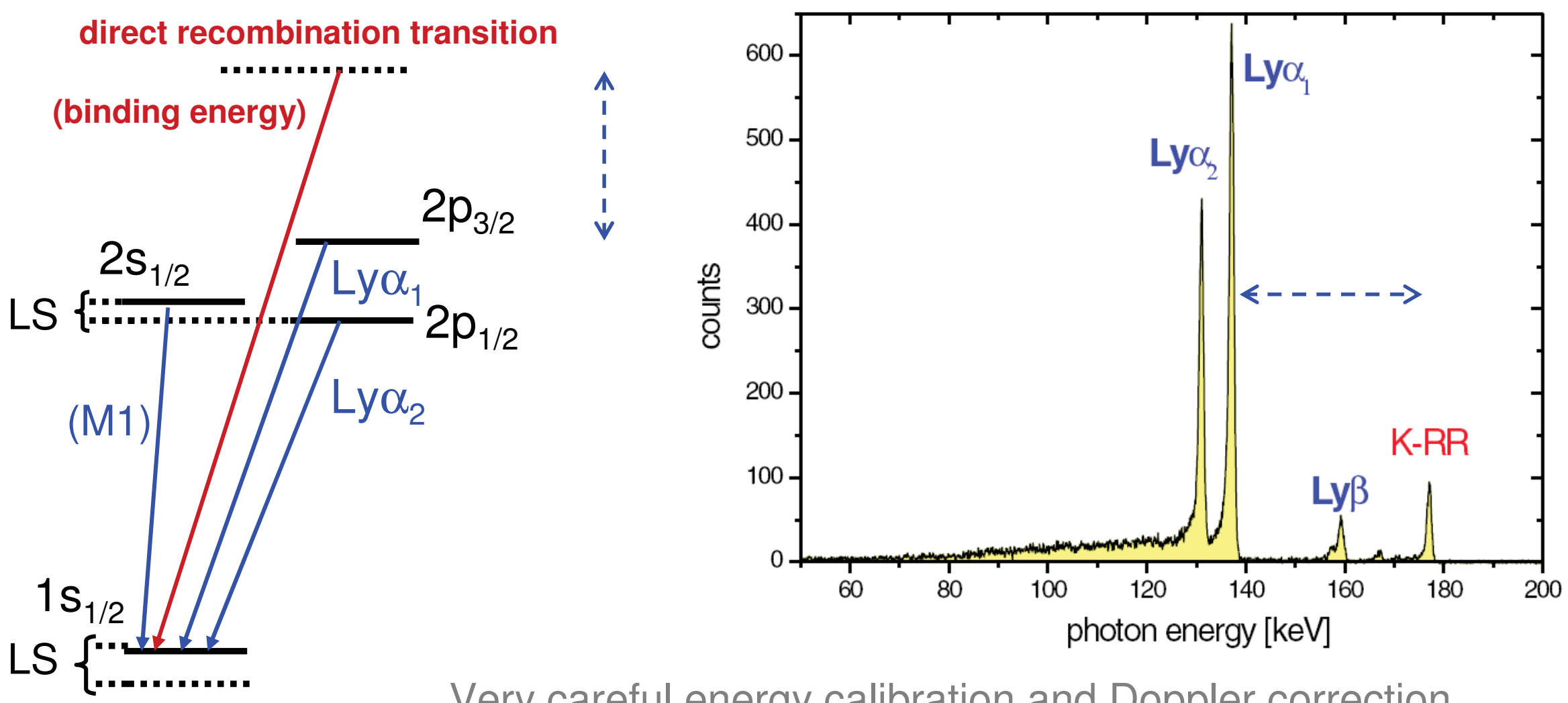

Very careful energy calibration and Doppler correction

A. Gumberidze et al., Phys. Rev. Lett. 94, 223001 (2005)

Result : $L_{1 S}=459.8(4.6) \mathrm{eV}$

Theoretical prediction : $463.95 \mathrm{eV}$ 


\section{Lamb shift in Li-like ions}

\section{Li-like ion : $3 \mathrm{e}^{-}$}

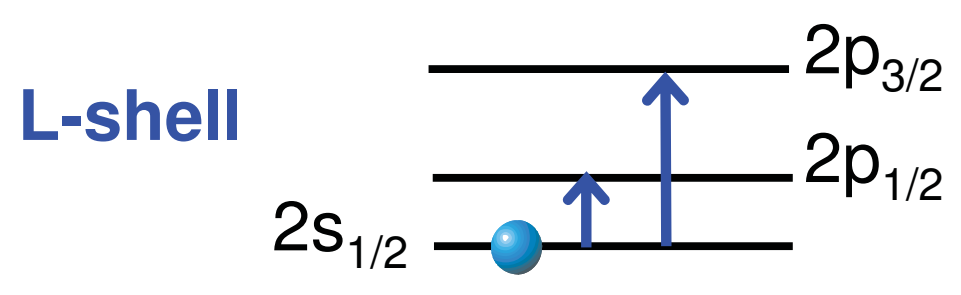

- simple theoretical description : only 1 valence electron

$\rightarrow$ small contributions from $\mathrm{e}^{-}-\mathrm{e}^{-}$interaction

- intra-shell transitions $(\Delta \mathrm{n}=0)$

$\rightarrow$ lower energy

Comparison of $\mathrm{H}$-like and Li-like data are required

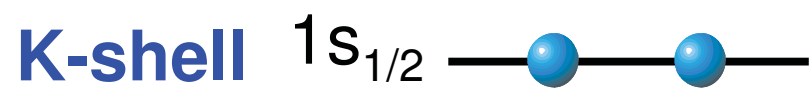

to disentangle QED and nuclear effects (Shabaev, Jentschura, Glazov, Yerokhin, et al.)

The contribution of two-loop QED terms in $\mathrm{L}_{1 \mathrm{~S}}$ of $\mathrm{H}$-like uranium is $-1.27 \mathrm{eV}$. It cannot be directly tested since it is smaller than the experimental accuracy $(4.6 \mathrm{eV})$

The calculation of high order QED terms in Li-like ions is more complex but the experimental accuracy is more than one order of magnitude better than the estimated size of the two-loop corrections 


\section{Measurement of the $2 s_{1 / 2}-2 p_{1 / 2}$ splitting in Li-like $\mathrm{HCl}(1)$}

- Measurement performed at Livermore using the superEBIT electron beam ion trap

P. Beiersdorfer et al., Phys. Rev. Lett. 95, 233003 (2005)

- Extreme ultraviolet emission spectroscopy of the $2 s_{1 / 2}-2 p_{1 / 2}$ transition in $U^{89+}$

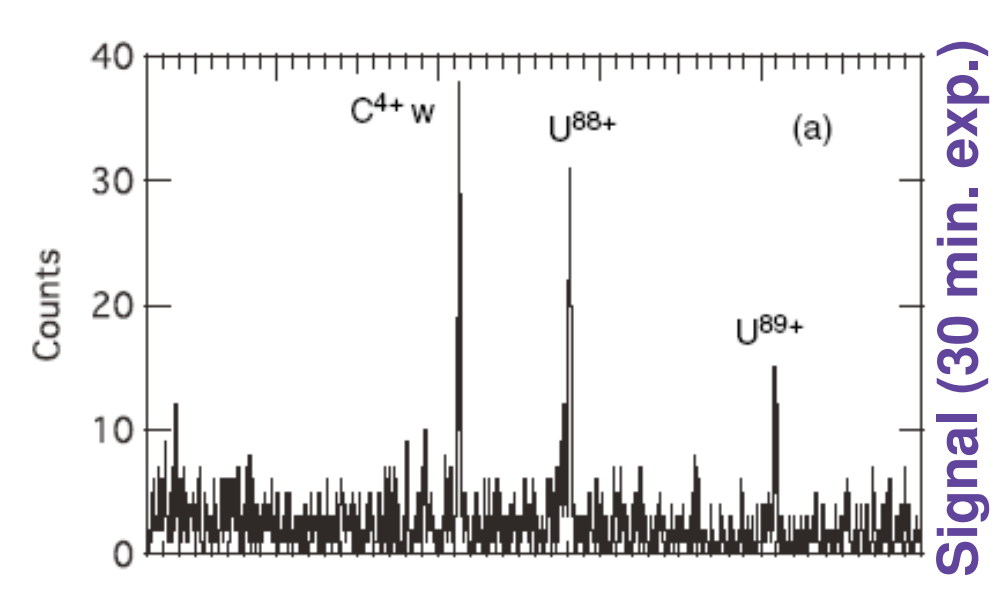
with a special grazing incidence spectrometer

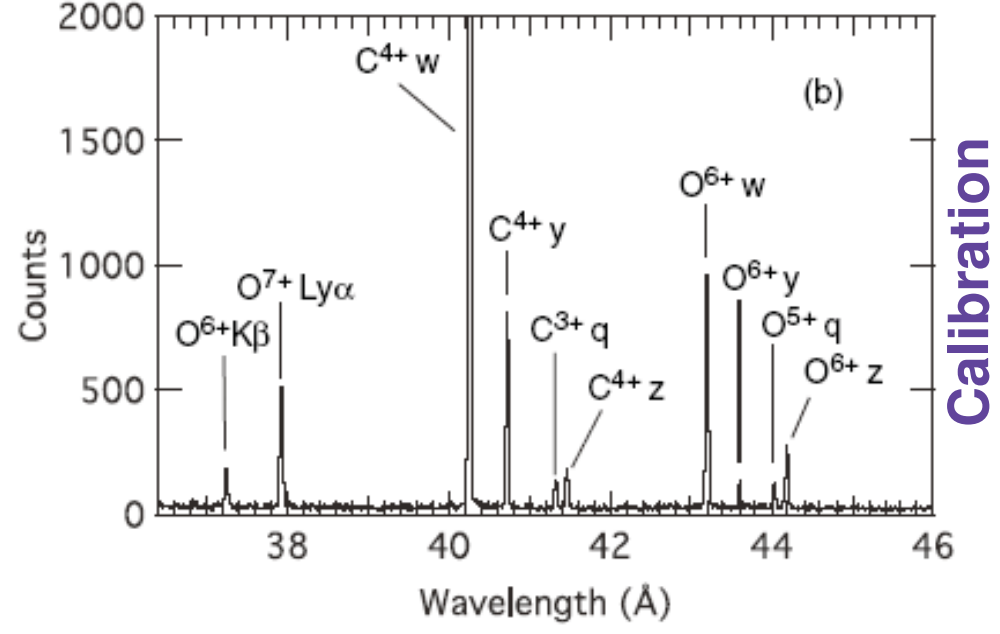

Result in Li-like $\mathrm{U}^{89+}: 280.645(0.015) \mathrm{eV}$

This result can be used to determine the two-loop Lamb shift in $U^{89+}$ since other contributions are well calculated

The value of the 1s two-loop Lamb shift in $\mathrm{H}$-like $\mathrm{U}^{91+}$ can been inferred : $-1.27 \mathrm{eV}$ in excellent agreement with the calculated value : $-1.26(0.33) \mathrm{eV}$

V.A. Yerokhin et al., X-Ray Spectrom. 32, 83 (2003) 


\section{Measurement of the $2 s_{1 / 2}-2 p_{1 / 2}$ splitting in Li-like $\mathrm{HCl}(2)$}

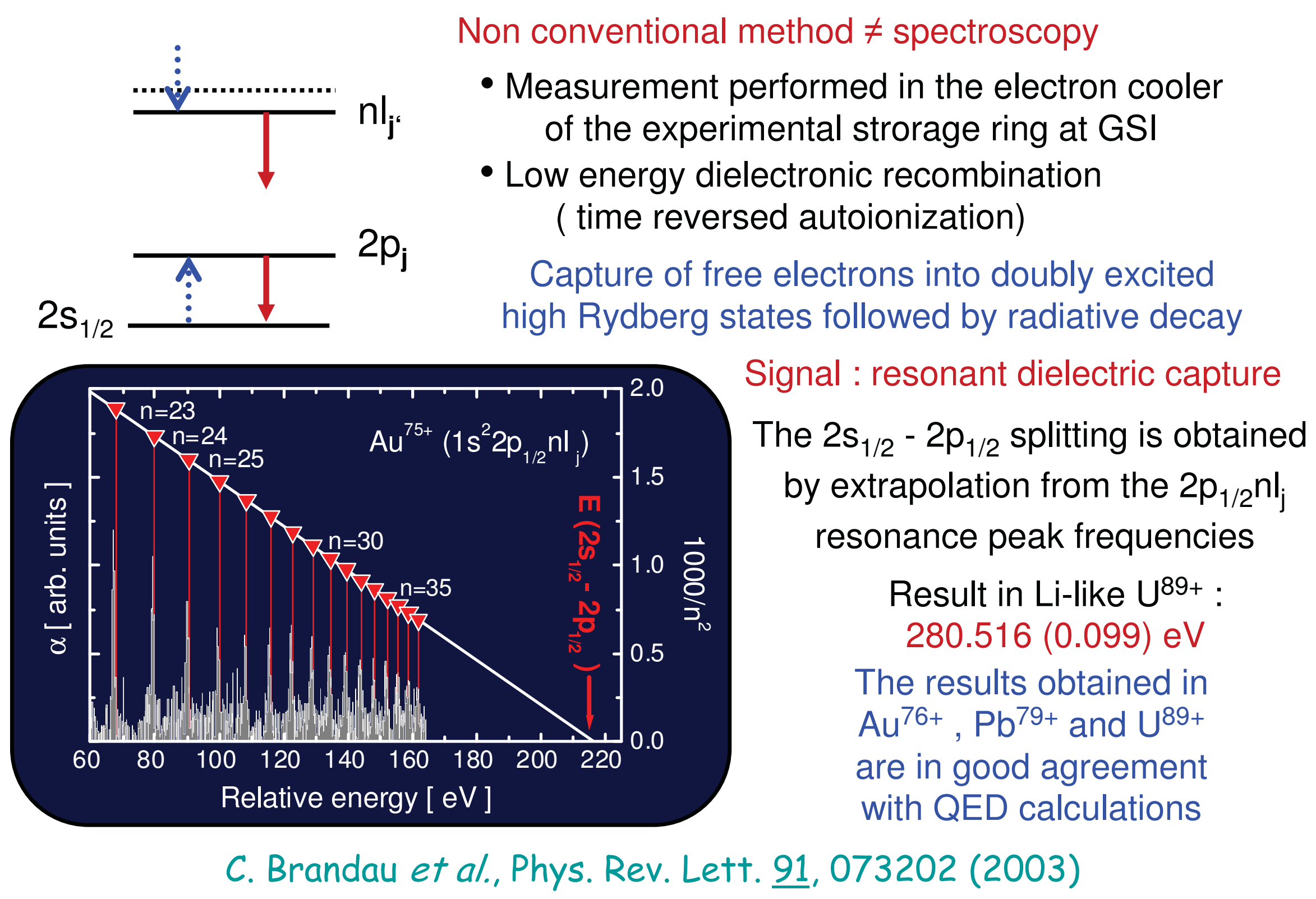




\section{Spectroscopy of highly charged He-like ions}

Heavy He-like ions

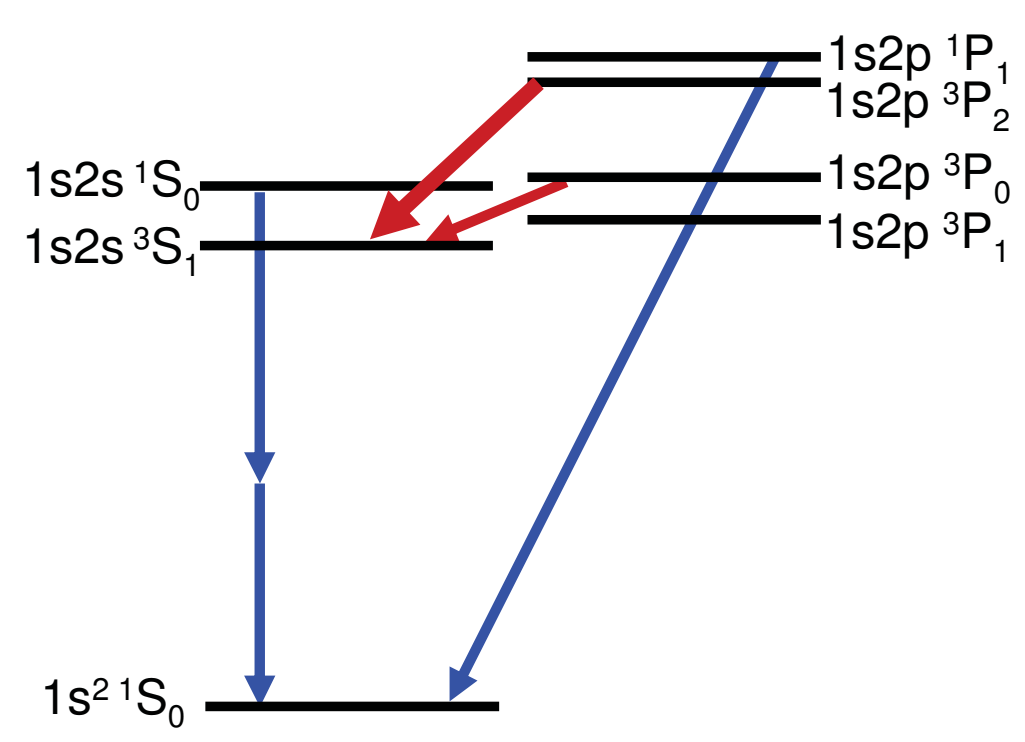

Heavy Li-like ions

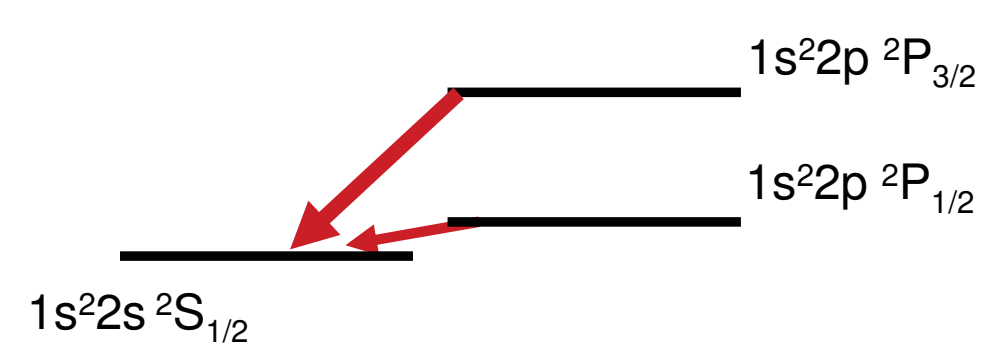

Intra-shell transition spectroscopy in He-like and Li-like ions

- same nucleus

- same one-electron QED contribution

$\rightarrow$ reduction of the systematic uncertainties

in the theoretical predictions 


\section{Spectroscopy of highly charged He-like ions}

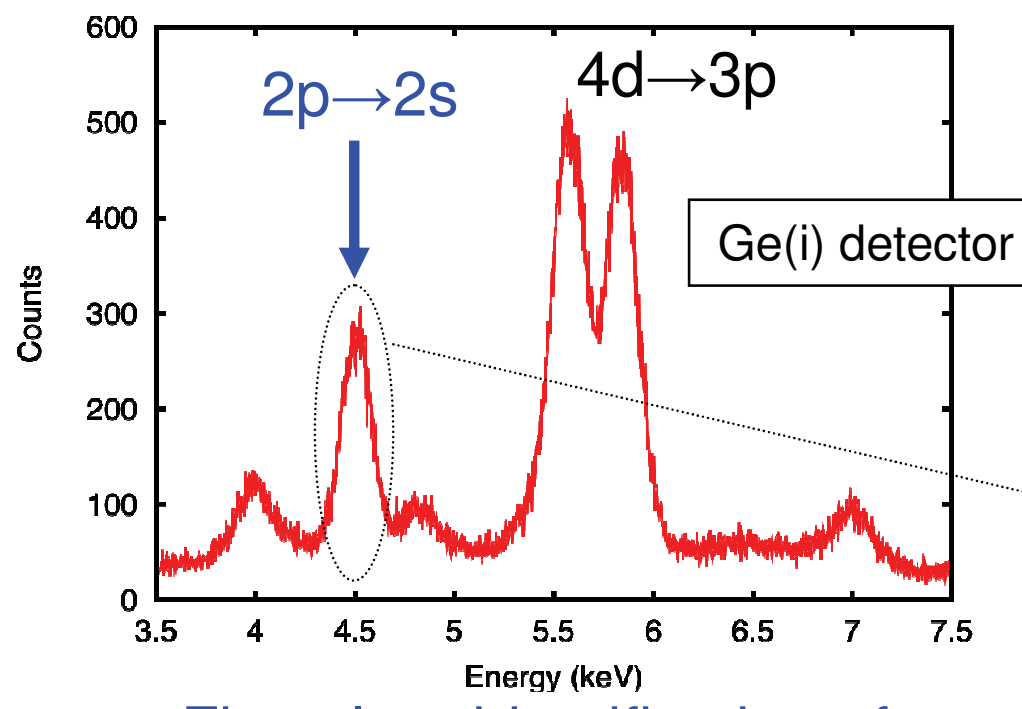

First clear identification of the $1 \mathrm{~s} 2 \mathrm{p}{ }^{3} \mathrm{P}_{2}-1 \mathrm{~s} 2 \mathrm{~s}^{3} \mathrm{~S}_{1}$ transition in He-like uranium

Precise measurement of the transition $\delta \mathrm{E} / \mathrm{E}=2 \times 10^{-4}$
- Measurement performed at the ESR in GSI

- Helium-like ions created by electron capture in a nitrogen gas jet target

- Detection with a Bragg crystal spectrometer (resolution few eV at 4-5 keV)

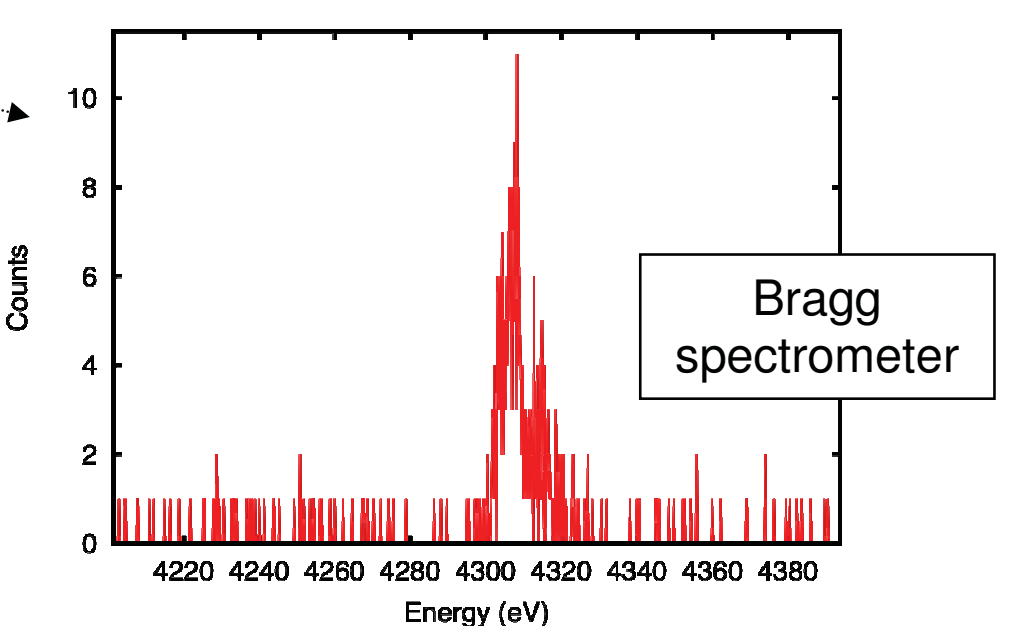

M. Trassinelli et al., Europhys. Lett. 87, 63001(2009)

result : $\quad 4509.71(0.99) \mathrm{eV}$

theory: $\quad 4509.86$ (0.07) eV Kozhedub and Shabaev, unpublished (2008) $4510.03 \quad$ eV Indelicato, unpublished (2008)

First test of two-electron QED terms in excited levels of heavy He-like ions 


\section{Spectroscopy of $\mathrm{HCl}$ : discussion}

QED predictions can be tested in heavy ions

- 1S Lamb shifts have been measured in H-like ions up to ${ }^{238} \mathrm{U}^{91+}$

They give a test of one-loop QED effects (all orders in Z $\alpha$ )

and are in agreement with theoretical predictions

- The $2 \mathrm{~s}_{1 / 2}-2 \mathrm{p}_{1 / 2}$ splitting determination in Li-like ions allows to derive the uncalculated two-loop contributions to the Lamb shifts

- Lamb shifts in He-like ions give a test of two-electron QED terms

Each type of these measurements provide a specific test of QED

Not discussed here : the hyperfine structure of $\mathrm{HCl}$

- Hyperfine splitting in $\mathrm{H}$-like ions up to ${ }^{209} \mathrm{Bi}^{82+}$ has been measured

Is not an efficient test of QED because of the nuclear magnetization

distribution correction (Bohr-Weisskopf) which is not well known

- Hyperfine splitting in Li-like ions combined with hfs of corresponding

$\mathrm{H}$-like ion is a way to overcome this limitation

Another QED test : the g-factor ! 